\title{
Le scritture dell'Italia preromana
}

\author{
The writing systems of \\ Pre-Roman Italy
}

\author{
Daniele Federico Maras \\ Soprintendenza Archeologia Belle Arti e \\ Paesaggio per l'area metropolitana di Roma, \\ la provincia di Viterbo e l'Etruria Meridionale \\ danielefederico.maras@beniculturali.it
}

Riassunto: La scrittura è stata introdotta nell'Italia antica attraverso il contatto tra i navigatori greci e le aristocrazie etrusche dell'età Orientalizzante. Entrando a far parte dei rapporti cerimoniali, si è diffusa presso le popolazioni vicine e nel corso del VI sec. a.C. ha assunto un valore identitario etnico, dando vita a un mosaico di tradizioni grafiche, volta per volta derivate direttamente dalla scrittura greca o attraverso la mediazione etrusca o latina. Lautore dedica alcune pagine introduttive al processo storico di trasmissione e adattamento della scrittura e poi passa in rassegna i domini epigrafici etrusco, falisco, latino, celtico, veneto, retico, camuno, paleo-italico, paleo-sabellico, ausone, osco e umbro. In conclusione, alcuni spunti per la ricerca futura vengono brevemente accennati.

Parole chiave: Epigrafia. Antica Italia. Etrusco. Sabellico. Sistemi di scrittura.

\begin{abstract}
Writing was introduced in ancient Italy through the contact between Greek sailors and Etruscan aristocracies in the Orientalizing period. As part of the ritual relationships, writing spread to the nearby peoples and in the course of the $6^{\text {th }} \mathrm{c}$. BCE became a marker of ethnic identity, thus creating a mosaic of graphic traditions, from time to time derived directly from Greek writing or through the Etruscan or Latin mediation. The chapter includes an introductory part on the historical process of transmission and adaptation of writing and a survey of the epigraphic domains of ancient Italy: Etruscan, Faliscan, Latin, Celtic, Venetic, Rhetian, Camunian, Paleo-Italic, Paleo-Sabellic, Ausonian, Oscan and Umbrian. In conclusion, some suggestions for future research are briefly presented.
\end{abstract}

Keywords: Epigraphy. Ancient Italy. Etruscan. Sabellian. Writing systems.

Recepción: 15.09.2019 | Aceptación: 12.03.2020 


\section{Introduzione generale}

Prima di affrontare la questione dell'introduzione, diffusione e sviluppo storico della scrittura in Italia, occorre chiarire alcuni concetti fondamentali, necessari alla comprensione degli argomenti trattati, per evitare equivoci e stabilire alcuni criteri di esposizione.

Va premesso che le definizioni che seguono derivano dalla riflessione in merito alla documentazione epigrafica dell'Italia pre-romana e sono pertanto funzionali alla comprensione dei concetti relativi a tale contesto culturale. La loro codificazione è stata impostata in modo generale, presupponendo la possibilità di applicazione dei medesimi concetti anche ad altri ambiti storici e culturali. ${ }^{1}$

Unità fondamentale della scrittura alfabetica è il segno alfabetico (o grafe$m a)$, per mezzo del quale i suoni della lingua sono rappresentati in una forma grafica, visibile e riproducibile. Si tratta di un elemento grafico composto di uno o più tratti - ed eventualmente di punti - combinati in una forma specifica, utilizzato come dato minimo per l'applicazione della scrittura, entro un insieme di elementi simili che compongono un modello alfabetico organizzato in una sequenza ordinata detta 'serie alfabetica'.

La caratteristica principale di un segno alfabetico è la sua distinguibilità dagli altri segni appartenenti al medesimo modello, necessaria al suo utilizzo nella rappresentazione dei suoni di una lingua, che non ammette la possibilità di equivoci.

In questo contesto, una variante è propriamente un allografo di un segno alfabetico, dal quale differisce solo per pochi tratti, mantenendo sostanzialmente una forma simile, utilizzato in alternativa per la rappresentazione del medesimo suono nell'applicazione della scrittura.

Esistono casi in cui un medesimo elemento grafico possa a seconda del contesto fungere da variante di due o più diversi segni alfabetici; in tal caso,

1 Per la terminologia e la relativa discussione metodologica si vedano in generale e senza pretesa di completezza, con riferimenti all'ampia bibliografia sull'argomento: Lejeune 1957; Cristofani 1969; Colonna 1970; Cristofani 1972; Colonna 1976; Bernardi Perini 1983; Johnston 1983; Harris 1989, 149-150; Briquel e Lejeune 1989, spec. 461-468; Cristofani 1990; Maggiani 1990; Pandolfini e Prosdocimi 1990, 158-160; Prosdocimi 1990, spec. 111-128 e 151-173; Cornell 1991; Blanck 1992, 12-27; Bagnasco Gianni 1999; Benelli 2004; Bagnasco Gianni 2008; Triantafillis 2008, 310-319; Maras 2009a, 309-311; Prosdocimi 2009; Maras 2015, 201-203; Maggiani 2018. 
ovviamente, nell'applicazione pratica l'uso dell'elemento per rappresentare un suono ne esclude la disponibilità per rappresentarne un altro.

Nell'insieme un modello alfabetico è il patrimonio di segni con le rispettive varianti da utilizzare per la trascrizione dei suoni di una lingua.

Un modello alfabetico non è necessariamente vincolato ad una sola lingua o cultura; tuttavia si tratta di un dato culturale fortemente caratterizzante e la sua adozione da parte di un gruppo umano, come componente della propria cultura materiale, è spesso indice di una precisa scelta di auto-rappresentazione, nella misura in cui la scrittura costituisce un elemento distintivo della propria identità culturale.

All'interno di un modello alfabetico viene operata in genere dagli scriventi una selezione dei segni e delle varianti, necessaria all'organizzazione di un proprio sistema scrittorio, che prelude a uno sviluppo autonoma del modello, che si distingue progressivamente da quello originario. Nel corso di questo processo è possibile che vengano introdotti nuovi segni o varianti e che la corrispondenza di segni e suoni venga modificata (anche fortemente) per le esigenze della lingua e della scrittura.

Dal concetto di modello alfabetico va distinto quello di serie alfabetica, che consiste in una sequenza teorica di segni disponibili per la rappresentazione dei suoni in un determinato contesto culturale e linguistico. Tale definizione, valida dal punto di vista epigrafico, può essere invertita dal punto di vista linguistico, considerando la serie alfabetica come la sequenza ordinata dei suoni rappresentati da segni alfabetici in un determinato contesto culturale.

Una serie alfabetica appartiene necessariamente ad un modello, del quale comprende l'intero patrimonio di segni, organizzato secondo una sequenza ordinata utile per l'apprendimento della scrittura, soprattutto per memorizzare la corrispondenza tra segni e suoni. In conseguenza di quest'uso mnemonico e tassonomico, la serie alfabetica acquista un valore numerico e garantisce ai singoli segni che la compongono la possibilità di essere usati in qualità di contrassegni numerali ovvero per marcare una sequenza di oggetti od elementi di cui si desidera conservare l'ordine.

Ancora diversa e più specifica è la definizione di alfabetario, vale a dire la rappresentazione grafica (in questo caso epigrafica) di una serie alfabetica, nella quale sono selezionate singole varianti di ciascun segno alfabetico, evidentemente ritenute più opportune o più corrispondenti al proprio uso grafico. Va sottolineato che spesso la serie alfabetica trascritta in un alfabe- 
tario non corrisponde a quella effettivamente utilizzata nella scrittura, né nel patrimonio dei segni, spesso più ampio, né nella selezione delle varianti.

Fin qui sono stati definiti gli elementi teorici e materiali necessari alla realizzazione pratica della scrittura; l'insieme degli usi grafici (a volte con forza di vere e proprie norme), che regolano l'applicazione della scrittura da parte di un gruppo umano per la rappresentazione dei suoni della propria lingua, viene definito sistema scrittorio.

Si tratta di un elemento imprescindibile per l'utilizzo della scrittura, di cui determina i criteri di applicazione e delimita le possibilità espressive, e la sua conoscenza è necessaria al lettore, destinatario del messaggio (epi)grafico, per comprenderne il contenuto.

Fanno parte di un sistema scrittorio: la selezione di segni e varianti del modello alfabetico di riferimento; l'attribuzione a ciascuno dei segni di un suono corrispondente; la creazione di nessi significativi, che esprimono suoni o combinazioni di suoni della lingua, altrimenti non rappresentate da singoli segni; la scelta di convenzioni grafiche riguardanti la direzione della scrittura, la sequenza di lettura delle righe, l'eventuale uso di segni di interpunzione, etc.

È importante osservare che a un medesimo modello alfabetico possono corrispondere diversi sistemi scrittori più o meno differenziati, sia per notare lingue diverse, sia per distinguere diverse 'province' scrittorie all'interno di una medesima comunità di scriventi.

Viceversa, di regola ad un diverso modello alfabetico corrisponde per necessità un sistema scrittorio differente, ma si osservano casi - soprattutto nel caso di dialetti affini o di prossimità culturale - in cui vengono adottate soluzioni simili e in contemporanea, che dimostrano la possibilità di interferenze e di fenomeni di imitazione dovuti all'influenza di un modello culturale più forte $\mathrm{o}$ prestigioso.

Resta infine da definire il concetto di riforma grafica, che si identifica nell'intervento di un'autorità istituzionale, ovvero di un individuo o di un gruppo dal prestigio culturale diffusamente riconosciuto, in merito ai criteri d'uso della scrittura da parte di una comunità: generalmente l'intervento sancisce una tendenza già in atto nella comunità degli scriventi, ma non mancano alcuni casi più o meno riusciti di riforme imposte dall'alto da un'autorità centrale.

Una riforma può determinare nuove norme grafiche vincolanti entro un sistema scrittorio, eventualmente per interrompere alcuni usi considerati 
inappropriati o per scegliere in modo definitivo entro uneccessiva varietà di soluzioni disponibili. L'intervento può altresì riguardare il modello alfabetico, dal quale possono essere cassati definitivamente alcuni segni ritenuti inutili oppure al quale possono essere aggiunti nuovi segni per venire incontro a nuove (o vecchie) necessità espressive finora non tenute in considerazione.

\subsection{Le scritture locali dell'Italia preromana: una definizione generale}

Nell'Italia antica, la scrittura è stata introdotta nell'ambito della cultura orientalizzante, a seguito del contatto dei navigatori e mercanti greci con le aristocrazie della regione tirrenica centrale. Di fatto, perciò, dal punto di vista tecnico e storico si tratta sempre e regolarmente di un adattamento dei modelli alfabetici greci, per quanto spesso attraverso alcuni passaggi di mediazioni culturali e rielaborazioni indipendenti di età storica. ${ }^{2}$

D’altro canto, la notevole varietà linguistica e il frazionamento etnico e culturale che caratterizzano la penisola italiana hanno favorito la diversificazione delle applicazioni della scrittura, con esiti a volte fortemente diversi nei diversi contesti storici e geografici. All'interrelazione storica delle scritture dell'Italia preromana sarà dedicato il paragrafo seguente, ma prima di questo vale la pena di offrire nelle prossime righe una definizione generale dell'argomento.

Il modello alfabetico greco introdotto inizialmente in Italia è stato quello euboico-calcidese degli ultimi decenni dell'VIII secolo a.C. È significativo, però, che oltre alle lettere d'uso nella scrittura greca (ivi compresi i segni complementari per $x i$, chi e phi) sia stata trasmessa alle popolazioni locali l'intera serie alfabetica teorica, comprendente anche le lettere abbandonate del modello alfabetico levantino (nella fattispecie le sibilanti samekh e tsade). Inoltre, la precoce presenza di alcune varianti grafiche non pertinenti alla scrittura euboica (quali il gamma semicircolare di tipo corinzio e il theta a circolo puntato di tipo ionico) prova la disponibilità di un patrimonio di segni più vasto a disposizione dei primi scribi tirrenici.

2 Sulla scrittura di età orientalizzante si vedano: Colonna 1970; Cristofani 1972; Colonna 1995; Bagnasco Gianni 1996; Riva 2006, 123; Wallace 2010; Wallace e Tuck 2011, 196-197; Maras 2013a, 331-340; 2014b; Pandolfini 2015; Maggiani 2018. Sulla prima alfabetizzazione in ambito greco e mediterraneo: Bartoněk e Büchner 1995; Lazzarini 1999; Ammirati, Biagetti e Radiciotti 2006. Per le più antiche attestazioni epigrafiche in Italia: Colonna 2005; Guzzo 2011; Camporeale 2015. 
Sembra evidente che la trasmissione della scrittura non sia stato un fenomeno occasionale e unicamente funzionale, ma abbia implicato la partecipazione diretta di 'maestri' professionisti, in possesso di competenze teoriche più ampie della mera conoscenza dei segni e della loro applicazione. ${ }^{3}$

Lalfabetario del Circolo degli Avori di Marsiliana d'Albegna, datato al secondo quarto del VII secolo a.C. (ET AV 9.1), prova l'esistenza di una sequenza teorica 'internazionale', in grado di trascrivere lingue diverse senza grandi trasformazioni: si tratta infatti sia della più antica serie alfabetica greca (euboico-calcidese) completa, sia di un documento dell'alfabetizzazione delle elites etrusco-meridionali. ${ }^{4}$

Per convenzione i nomi dei segni sono quelli dell'alfabeto greco, salvo le lettere non in uso e quello dell'aspirata, per le quali si fa ricorso ai nomi fenici; per pochi segni, infine, si preferisce una definizione descrittiva (come il segno a croce di S. Andrea e il tridente, corrispondenti rispettivamente a $x i$ e chi nell'uso euboico).

La sequenza strumentale comprende 26 segni, che nell'uso teorico greco sono così articolati:

- n. 5 vocali (alpha, epsilon, iota, omicron, ypsilon);

- n. 1 semivocale (digamma);

- n. 10 occlusive (sonore: beta, gamma, delta; sorde: kappa, pi, qoppa, tau; aspirate: theta, phi, chi);

- n. 1 aspirata (het);

- n. 2 nasali $(m y, n y)$;

- n. 2 liquide (lambda, rho);

- n. 5 sibilanti (semplice: sigma; complesse: zeta, xi; non in uso: samekh, tsade);

Come è naturale, la diversa fonologia delle lingue dell'Italia ha comportato una diversa selezione delle lettere da utilizzare nell'applicazione pratica della scrittura, come nel caso della lingua etrusca (priva delle occlusive sonore e della vocale velare aperta /o/) o di diverse lingue italiche (prive delle occlusive aspirate). Al contrario, l'esistenza di suoni non rappresentati nel modello

3 Pandolfini e Prosdocimi 1990, 164-165 e 195-196; Maras 2014b, 104-106.

4 Pandolfini e Prosdocimi 1990, 19-21, n. I.1, e 195-205; Bagnasco Gianni 1996, 225-227, n. 221. Vedi anche Colonna 1976, 7-11; Prosdocimi 1990, 159-161. 
greco ha comportato nel corso del tempo l'introduzione di ulteriori segni, di regola aggiunti in fondo alla serie alfabetica, come nel caso delle diverse soluzioni per la spirante labio-dentale $/ f /$ e per le vocali intermedie italiche, trascritte convenzionalmente come $<i<$ e $<\dot{u}>$.

Attraverso vari passaggi e adattamenti della serie alfabetica, che riflettono le caratteristiche dei diversi sistemi scrittori (con operazioni di selezione, introduzione di diacritici e vere e proprie riforme grafiche), dal primo modello alfabetico unitario dell'età orientalizzante si arriva nel corso dei secoli alle diverse scritture locali dell'Italia preromana, a volte articolate in varianti regionali o persino cittadine. In questo contesto, nel prosieguo di questo capitolo, si manterrà la distinzione tra scritture derivate direttamente dal modello greco (p. es. etrusca, enotria, ausone, messapica, sicula, elima e lucana), scritture mediate dal modello etrusco (p. es. latina, falisca, leponzia, paleo-italiche, camuna, retica, veneta, umbra, pre-sannitica, osca) e scritture mediate dal modello latino (p. es. ernica, peligna, vestina, marrucina, volsca, tardo-umbra e tardo-lucana). ${ }^{5}$

\subsection{Origini e sviluppo storico delle scritture dell'Italia preromana}

Fatta eccezione per l'antichissima e isolata iscrizione di Osteria dell'Osa, presso l'antica città di Gabii, la cui attribuzione e interpretazione sono ancora oggetto di discussione, ${ }^{6}$ le più antiche attestazioni note della scrittura in Italia risalgono al periodo tra la seconda metà dell'VIII e l'inizio del VII secolo a.C. e si riferiscono alla lingua etrusca. Si tratta di iscrizioni appartenti a due categorie fondamentali: A) testi di dono tra membri delle élites aristocratiche; B) lettere isolate e contrassegni di produzione utilizzati dagli artigiani. Nella prima categoria rientrano in verità anche alcune iscrizioni onomastiche, il cui scopo era quello di registrare il nome del destinatario di un dono. ${ }^{7}$

La stretta selezione dei contesti culturali in cui compare inizialmente la scrittura è indicativa dell'ambiente entro il quale essa si è diffusa, nel più ampio alveo della cultura Orientalizzante mediterranea. Infatti, come avvenne per l'ideologia del banchetto aristocratico, per il consumo cerimoniale del vino e per la circolazione di elementi iconografici e tecnologie, anche la scrittura è giunta in Italia a partire da un modello vicino orientale, attraverso la

5 Colonna 1988a; Briquel e Lejeune 1989, spec. 468-471; Rocca 2000; Maras 2017, 63-67.

6 Colonna 2005; Guzzo 2011; Maras 2015, 201-202.

7 Cristofani 1982; Colonna 1988b; Maras 2015, 201-206; 2017, 63-64; 2020. 
mediazione greca, e ha interessato in un primo momento soprattutto le élites e gli artigiani che operavano al loro servizio. In questo contesto lo scriba non era altro che una delle diverse figure tecniche specializzate delle 'corti' aristocratiche e in generale gli artigiani (p. es. ceramisti, bronzisti, intagliatori e tessitori/trici) ebbero precocemente accesso all'alfabetizzazione per venire incontro alle richieste che spesso ricevevano dai propri committenti, al cui servizio lavoravano. ${ }^{8}$

In questo contesto, lapplicazione della scrittura non era legata a uno specifico ambito etnico o linguistico, quanto piuttosto al più elevato ambito sociale e, con l'ulteriore estensione della rete gli scambi e i contatti tra le élites della penisola italiana, la conoscenza dell'alfabeto venne trasmessa alle popolazioni contermini, sia all'interno della compagine etrusca (verso nord, a Vetulonia, Chiusi e in Etruria Padana), sia oltre Tevere (presso i Latini e i popoli italici), sia nell'Italia settentrionale presso i Celti dell'area Golasecchiana.

L'introduzione della scrittura in Etruria settentrionale risale alla metà del VII secolo a.C., quando sono note le iscrizioni di dono incise su alcuni kyathoi di bucchero di produzione vetuloniese a decorazione mista (incisa, excisa e a rilievo) e su una situla d'argento da Chiusi (ET Cl 2.1-2). In entrambi i casi è stato sottolineato il rapporto con l'ambiente artigianale di Caere, che dimostra quali siano stati i canali preferenziali per la diffusione delle pratiche scrittorie. ${ }^{9} \mathrm{Va}$ invece forse riferita a una diversa tradizione la fibula di Castelluccio (ET Cl 2.3), se può essere accolta la recente proposta di una sua cronologia alta già nel secondo quarto del VII secolo a.C. ${ }^{10}$

Poco più tardi, tra la seconda metà del secolo e l'inizio del successivo, si datano iscrizioni di area fiesolana (il cd. 'incensiere' di Artimino e le kotylai di Castelnuovo Berardenga; ET Fs 6.1 e AS 3.1-2) e padana (i cippi di Rubiera ET Pa 1.1-2), nonché, scavalcando la valle del Po, le prime attestazioni epigrafiche celtiche di area Golasecchiana (a Sesto Calende e Castelletto Ticino). Quanto a queste ultime, è significativo che alla fonologia celtica sia stato adattato non già il modello alfabetico completo di tradizione greca, ma il modello d'uso della neonata scrittura etrusca settentrionale, privo dei segni per le occlusive sonore, ma che conservava ancora il segno per la vocale $/ o / .{ }^{11}$

8 Maras 2012a; Tuck e Wallace 2020; Maras c.s.

9 Discussione e bibliografia in Bagnasco Gianni 1993; 2008, 53-56; Maras 2013a, 333-339; Maggiani 2014; Cappuccini 2018.

10 Maggiani 2018.

11 Maras 2014a; 2014b. 
Per quanto riguarda le modalità della trasmissione della scrittura, leccezionale testimonianza di un bicchere d'impasto di produzione locale da una tomba a fossa della necropoli di via Sculati a Sesto Calende ha conservato la doppia iscrizione di uno "scriba" etrusco $(z i \chi u)$ e di un individuo locale, che presumibilmente intrattenevano un rapporto di maestro e allievo nell'ambito di uno scriptorium locale. ${ }^{12}$

Pressoché contemporanea è l'adozione della scrittura etrusca da parte dei popoli del Lazio, a partire dal modello completo originario, con una diversa selezione dei segni d'uso: la mediazione etrusca è evidente nell'utilizzo del segno del gamma per la velare sorda anziché sonora. Le più antiche testimonianze in questo senso si datano verso la fine del VII secolo a.C. e riguardano la lingua latina (a Roma e a Satricum) ${ }^{13}$ e quella ernica (ad Anagni). ${ }^{14}$

A nord del Tevere, le enclaves culturali della regione falisca e di Capena (rispettivamente di lingua latina e italica) sembrano aver partecipato direttamente al fenomeno scrittorio dell'Etruria meridionale, come è dimostrato da diverse attestazioni di pieno VII secolo a.C., in cui si osserva la precoce introduzione di nuove varianti e segni come lo het a rettangolo vuoto e il segno a freccia (il secondo dei quali usato per la spirante labiodentale $/ f /$ ). ${ }^{15}$

Il primo di questi due segni ebbe fortuna nelle scritture paleo-italiche della regione tiberina, attestate a partire dall'inizio del VI secolo a.C. per trascrivere alcune varianti linguistiche italiche orientali di ceppo umbro-sabino (o, più propriamente, 'safino'). ${ }^{16}$ Benché derivate dal modello etrusco, queste grafie presentano unalta variabilità nella forma e disposizione delle lettere $\mathrm{e}$ vedono l'introduzione di ulteriori segni: in particolare, i segni a cancelletto (samekh) e a tridente (chi), inutili per la fonologia umbro-sabina, sono stati 'ripescati' e convertiti per marcare rispettivamente le vocali intermedie $<i>$ e $<\dot{u}>$, mentre il suono $/ f /$ è reso per la prima volta dal segno a $<8>$, poi diffuso in molti modelli alfabetici dell'Italia centrale e meridionale.

12 Maras 2012a; 2014b.

13 Colonna 1980, 63, n. 29; 2014; Mancini 2004, 22-23; Hartmann 2005, 25-26; Maras 2009c, 435, n. A.3.

14 Colonna e Gatti 1992, 320, n. 1; Attenni e Maras 2004, 75-78.

15 Maras 2013b, 269-270.

16 De Simone 1992; Rix 1996; Rocca 1997; 2000, 179-186; Colonna 2001; Minetti 2008; Triantafillis 2008, 289-304; Agostiniani et alii 2011, 8-21; Crawford 2011; Maras 2012b; Morandi 2017, 103-113. 
Nel corso del VI secolo, le diverse scritture assumono sempre più un valore identitario etnico, fatto che ha come conseguenza la selezione intenzionale di forme e varianti grafiche differenziate rispetto ai modelli alfabetici delle regioni vicine. Tale fenomeno è particolarmente evidente nel caso della scrittura paleo-sabellica dell'area sud-picena (a partire dall'iscrizione del Guerriero di Capestrano, ST Sp AQ 2) ${ }^{17}$ e in quella ausone della penisola sorrentina (cd. 'alfabeto nocerino'), ${ }^{18}$ entrambe diffuse tra VI e V secolo a.C. Nel primo caso la grafia assume forme quadrangolari e seleziona varianti speciali, con circoli ridotti a punti (specialmente la $<0>$ e il segno a $<8>$ ), che rendono la scrittura fortemente diversa dalle altre dell'Italia centrale. Nel caso dell' 'alfabeto nocerino, invece, il modello di partenza è quello greco di Cuma, ma molte lettere appaiono ruotate di $90^{\circ} \mathrm{o} 180^{\circ}$ e per marcare il suono $/ \mathrm{s} /$ è stato selezionato un segno ad alberello che recupera il valore di sibilante del samekh fenicio. Altri segni peculiari sono il digamma uncinato, che rende la / $f$ / (come nella grafia enotria del cippo del Tortora, ST Ps 20), e lo iota diacriticato per la vocale intermedia $<i<$.

Nonostante la breve durata e la distribuzione relativamente limitata delle attestazioni, alcune delle innovazioni legate a queste scritture hanno avuto seguito in modelli alfabetici più recenti, come la forma quadrangolare delle lettere, il segno a $<8>$ e lo iota diacriticato nellosco, ovvero il digamma diacriticato nella grafia enotria del cippo del Tortora (ma anche nella scrittura paleo-sabellica come variante del normale digamma). ${ }^{19}$

In realtà, al di là della scarsa documentazione - dellordine di alcune decine di iscrizioni e un certo numero di segni isolati - è possibile intravedere una certa comunità culturale tra gli ambienti scrittori della costa tirrenica centro-meridionale e l'area italica centrale e orientale in età tardo-arcaica, attraverso la quale alcuni segni e riforme grafiche vengono condivisi, sotto l'influenza costante del sistema scrittorio etrusco, particolarmente evidente nelle cosiddette iscrizioni pre-sannitiche della Campania e del Lazio meridionale..$^{20} \mathrm{Da}$ questa comunità prese le mosse nel IV secolo a.C. la scrittura nazionale osca, che assunse poi una forma sistematica e venne utilizzata in

17 Marinetti 1985; Rocca 2000, 182-183; Calderini, Neri e Ruggeri 2007; Triantafillis 2008, 237-289; La Regina 2010; Agostiniani et al. 2011, 92-93.

18 Colonna 1994; Russo 2005, 27-81; Agostiniani 2010; Poccetti 2010.

19 Lazzarini e Poccetti 2001.

20 Colonna 1992; Cristofani 1996; Russo 2005, 85-99; Tagliamonte 2010; Calderini 2012; Maras 2012c. 
modo pressoché regolare fino alla definitiva diffusione della scrittura latina. ${ }^{21}$ Caratteristiche di questo sistema sono l'assenza della /o/ (alla maniera etrusca) e l'uso dei segni iota e ypsilon diacriticati per le vocali intermedie $<i ́$ e $<u ́$. La presenza dei segni per le occlusive sonore dipende probabilmente dall'ascendenza ausone (nocerina) del modello alfabetico, sul quale sono stati innestati elementi delle scritture pre-sannitiche, a loro volta improntate al modello etrusco.

In Italia settentrionale, accanto alla scrittura golasecchiana, si sviluppa a partire almeno dalla metà del VI secolo a.C. il dominio epigrafico veneto, che in questa prima fase fa uso di un modello alfabetico etrusco di tradizione chiusina. Anche in questo caso, come per la lingua celtica, l'adattamento fonologico della scrittura portò alla scelta di trasporre le due serie di occlusive etrusche (sorde-aspirate) nelle due serie di occlusive venete (sorde-sonore), con esiti complessi e non sempre regolari (p. es. nell'iscrizione del kantharos di Lozzo Atestino, tau $\sim / d /$ e theta $\sim / t /$, ma $c h i \sim / g /) .{ }^{22}$

Lesistenza di una scuola scrittoria in relazione con il santuario della dea Reitia a Este portò già a partire dagli ultimi decenni del VI secolo all'introduzione di un nuovo sistema scrittorio basato su un diverso modello alfabetico, caratterizzato da forme quadrangolari e linee oblique e dall'impiego regolare dell'interpunzione sillabica di tradizione etrusco-meridionale. Questo secondo sistema, pur attraversando alcuni sviluppi interni e riforme grafiche, mantiene la propria stabilità e regolarità fino alla romanizzazione. ${ }^{23}$

Anche nel caso della scrittura retica, il modello di partenza è stato quello etrusco settentrionale, ma in questo caso l'adattamento fonologico è stato più semplice a causa dell'affinità delle due lingue, appartenenti al medesimo gruppo "tirsenico". ${ }^{24}$ Va notata però la necessità sentita dagli scribi retici di introdurre un diverso segno per una dentale marcata (convenzionalmente trascritta come $\left\langle t^{\prime}\right\rangle$ ) al posto del theta, che pure rimaneva inutilizzato. In base alla forma che assume questo segno complementare si distinguono i due

21 Poccetti 1979; Colonna 1984; Rocca 2000, 190-192; Cooley 2002; Wallace 2004a; Triantafillis 2008, 81-142; Muscariello 2015.

22 Lejeune 1974, 243-246, n. 123; D. Locatelli e A. Marinetti, in Marinetti 2002, 157-158, n. 1; si vedano anche Pellegrini e Prosdocimi 1967; Prosdocimi 2007; Wallace 2004b.

23 Colonna 1988a, 143-147; Prosdocimi 2007.

24 Schumacher 1992; 1998; 2004; Rix 1998a; Morandi 1999; De Simone e Marchesini 2014; Marchesini 2014; 2015; Salomon 2017. 
gruppi epigrafici distinti di Magrè (in cui il segno assume la forma di un beta con tre occhielli) e di Sanzeno (dove si usa un segno a freccia).

Più difficile, invece, è ricostruire lo sviluppo storico più antico della scrittura camuna, attestata soltanto da incisioni rupestri che, in quanto tali, restano prive di un contesto archeologico in grado di fornire indicazioni cronologiche precise. ${ }^{25} \mathrm{~A}$ differenza delle altre scritture dell'Italia settentrionale lalfabeto camuno conserva la serie completa delle occlusive sonore, della /o/ e di altri segni, che pure mancano nella scrittura d'uso, come documentato da alcuni alfabetari incisi a Piancogno. ${ }^{26}$ Inoltre, la scrittura fa uso di un segno ad alberello per la sibilante, presumibilmente derivato dal samekh o dalla zeta, ma usato come variante del sigma nella sequenza alfabetica e sostanzialmente identico per forma e funzione all'analogo segno della scrittura ausone. Alla medesima tradizione sembra accostarsi inoltre la forma del $p i$, che riprende il digamma uncinato delle scritture ausone e paleo-sabellica.

Si direbbe pertanto che il canale di trasmissione di questa scrittura sia stato diverso da quello etrusco settentrionale tardo-orientalizzante, con contatti italici centrali e meridionali, anche se allo stato attuale non sembra possibile determinarne con certezza la natura.

Un'ultima nota merita la notevole somiglianza tra il modello alfabetico camuno e le più antiche forme delle rune germaniche, risalenti ai primi secoli dell'era cristiana, che documenta una innegabile interdipendenza dei due sistemi al di là della distanza di tempo e di spazio. ${ }^{27}$ E possibile pertanto ricostruire in linea teorica una tradizione grafica quasi interamente perduta, che ha trasmesso la scrittura etrusco-italica oltre l'arco alpino ed è sopravvissuta alla diffusione della scrittura latina fino a riaffiorare in documenti tardo-antichi e medievali.

26 Tibiletti Bruno 1990, 67-76; Morandi 2017, 450, n. 628.

27 Rix 1992; Stoklund 1997; Morandi 2017, 457-459. 


\section{Le scritture alfabetiche dell'Italia preromana}

\subsection{Scrittura etrusca}

Scrittura alfabetica derivante dal modello euboico-calcidese. ${ }^{28}$ Inizialmente venne trasmessa lintera sequenza alfabetica teorica, completa anche dei segni che non erano in uso nella scrittura greca e dei segni complementari aggiunti alla fine (circolo tagliato, tridente e croce di S. Andrea, rispettivamente utilizzati per $p h i$, chi e $x i$ nel modello calcidese). Il numero dei caratteri, pertanto, come documentato dall'alfabetario di Marsiliana d'Albegna (ET AV 9.1, secondo quarto del VII secolo a.C.) comprendeva 26 segni.

La prima acquisizione della scrittura interessò le metropoli dell'Etruria meridionale, dove sono attestati esempi di scrittura già alla fine dell'VIII secolo a.C. (a Tarquinia, Veio e Vulci; solo al principio del secolo seguente a Cerveteri e più a nord a Bologna).

Ladattamento alla lingua etrusca comportò una diversa selezione dei segni d'uso, con l'abbandono dei segni delle occlusive sonore beta e delta, dell'omicron e delle sibilanti superflue samekh e tsade, nonché del segno a croce. Questi segni, però, conservarono la propria posizione nella sequenza alfabetica almeno fino alla metà del VI secolo a.C.

L'introduzione di norme grafiche consentì di risolvere il problema di esprimere la spirante labiodentale /f/ con un digrafo $(h v$ o $v h)$ e di introdurre una ortografia delle velari con gamma di fronte ai suoni palatali /e/e /i/, qoppa di fronte al suono $/ u /$ (in funzione sia di vocale velare chiusa, sia di approssimante labio-velare sonora) e kappa di fronte alla vocale aperta /a/.

Attorno alla metà del VII secolo a.C. o poco prima è attestato l'uso della scrittura nei centri dell'Etruria settentrionale, a partire da Vetulonia e Chiusi, in una variante depurata dei segni non utilizzati, che assegnava al solo kappa la funzione di velare sorda e introduceva l'opposizione di tsade e sigma, rispettivamente per la sibilante semplice e marcata (quest'ultima in funzione di fricativa palatale).

Circa un secolo dopo anche la scrittura etrusco-meridionale recepì l'opposizione funzionale delle sibilanti, ma con l'esito inverso di usare il sigma per

28 Bibliografia di riferimento: Colonna 1970; 1976; 1980; Cristofani 1972; Roncalli 1985; Maggiani 1990; Bagnasco Gianni 2000a; 2000b; Rix 2000; Hartmann 2005; Wallace 2008, spec. 17-28; 2010; Maras 2012a; 2015; 2020; Bruschetti et al. 2015; Moandi 2017, 23-66; Maggiani 2018. 
la /s/ semplice e il tsade per la /ś/ marcata; quanto alla velare, la scelta ricadde sul solo gamma, determinando così due distretti grafici maggiori nella regione etruscofona. Infine, verso la fine del VI secolo, la grafia di Cerveteri scelse di ruotare il tsade di $90^{\circ}$, così trasformandolo nel cd. sigma a quattro tratti che rimase tipico di tale città. ${ }^{29}$

Sempre nei decenni finali del VI secolo, venne introdotto in Etruria il segno a $<8>$, aggiunto in fondo alla sequenza alfabetica, per marcare la spirante labiodentale $/ f /$, così risolvendo finalmente la carenza fonologica del modello greco.

Da questo momento, fino alla definitiva adozione della lingua e scrittura latina nel corso del I secolo a.C. la sequenza alfabetica rimase piuttosto stabile, salvo varianti locali e legate a usi grafici specifici, come il my a V capovolta e l'epsilon rovesciato di Cortona o la $<0>$ bicaudata della bilingue di Pesaro.

\subsubsection{Tavola dei caratteri}

1. Alfabetario di Marsiliana d'Albegna (secondo quarto del VII sec. a.C.)

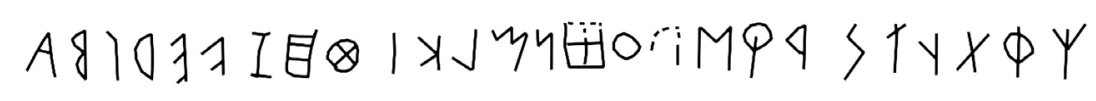

2. Modello alfabetico d'uso orientalizzante (VII sec. a.C.)

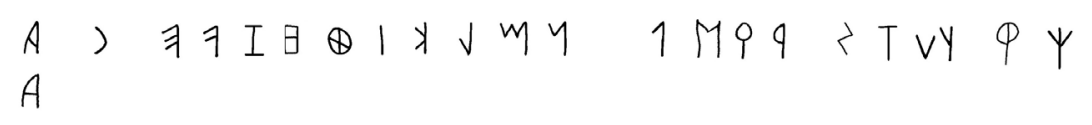

3. Modello alfabetico d'uso dei kyathoi di Caere-Vetulonia (metà VII sec. a.C.)
$A$
$A$$\exists \exists$
$\begin{array}{ccccc}0 & 1 & 1 & J & y \\ 0 & \neq & m\end{array}$
$1 M 99 \leqslant T V$
$\checkmark$
A

4. Modello alfabetico settrentrionale arcaico (VI sec. a.C.)
A

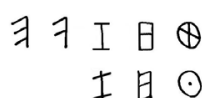
$1 M$
$q<\uparrow \vee \phi \psi$
$\mathrm{m} \mathrm{n}$

5. Modello alfabetico merionale arcaico (VI-V sec. a.C.)
A $>$
$\exists 习 I$
士日 $\odot$
$1 M 990<+v$
$\phi \% 8$ 
6. Modello alfabetico ceretano tardo-arcaico (fine VI-V sec. a.C.)

$$
A>\exists 7 \pm \theta \odot 1 \times \sqrt{ } \text { ) }
$$

7. Modello alfabetico d'uso di età recente (IV-I sec. a.C.)

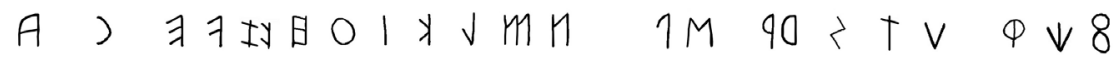

Trascrizione:

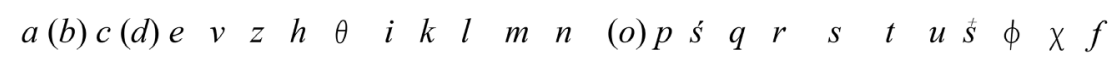

Val. fonetico meridionale:

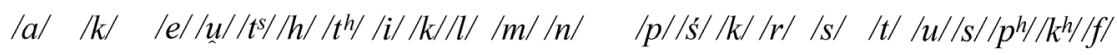

Settentrionale:

$$
|s /| s /
$$

\subsubsection{Esempi illustrativi}

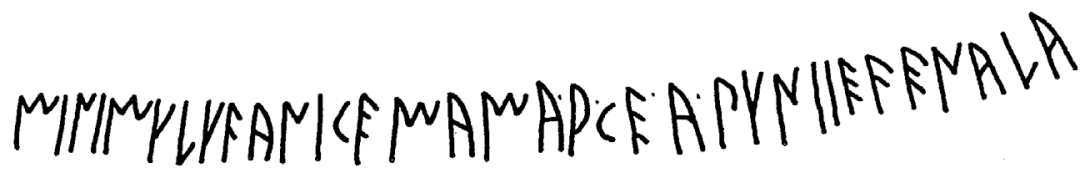

Fig. 1. Veio, santuario di Portonaccio. Oinochoe di bucchero (fine del VII sec. a.C.) mini muluvanice mama.r.ce a.puniie venala

Bibl.: Colonna 1987, 427-428; CIE 6421; Maras 2009d, 410, Ve do.3; ET Ve 3.5.

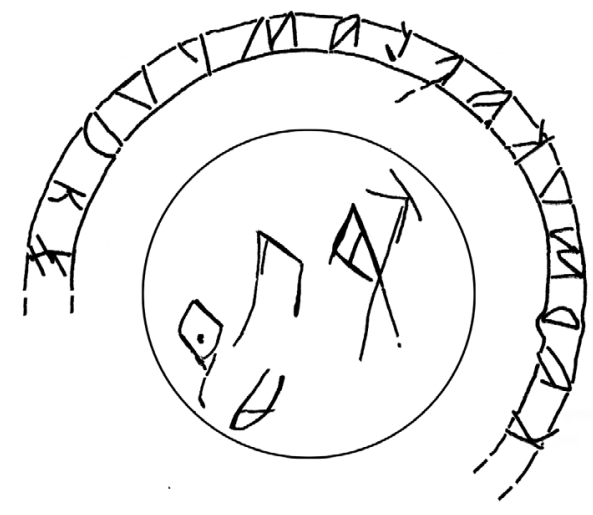

Fig. 2. Populonia, necropoli di San Cerbone. Glaux attica a figure rosse (metà del V sec. a.C.) ${ }^{a}$ kav $\theta / a$ b karmu kavzaś turke Bibl.: D. F. Maras, in REE LXIII, 1997, n. 38; Maras 2009d, 330-332, Po do.1; ET Po 3.2+4.2. 


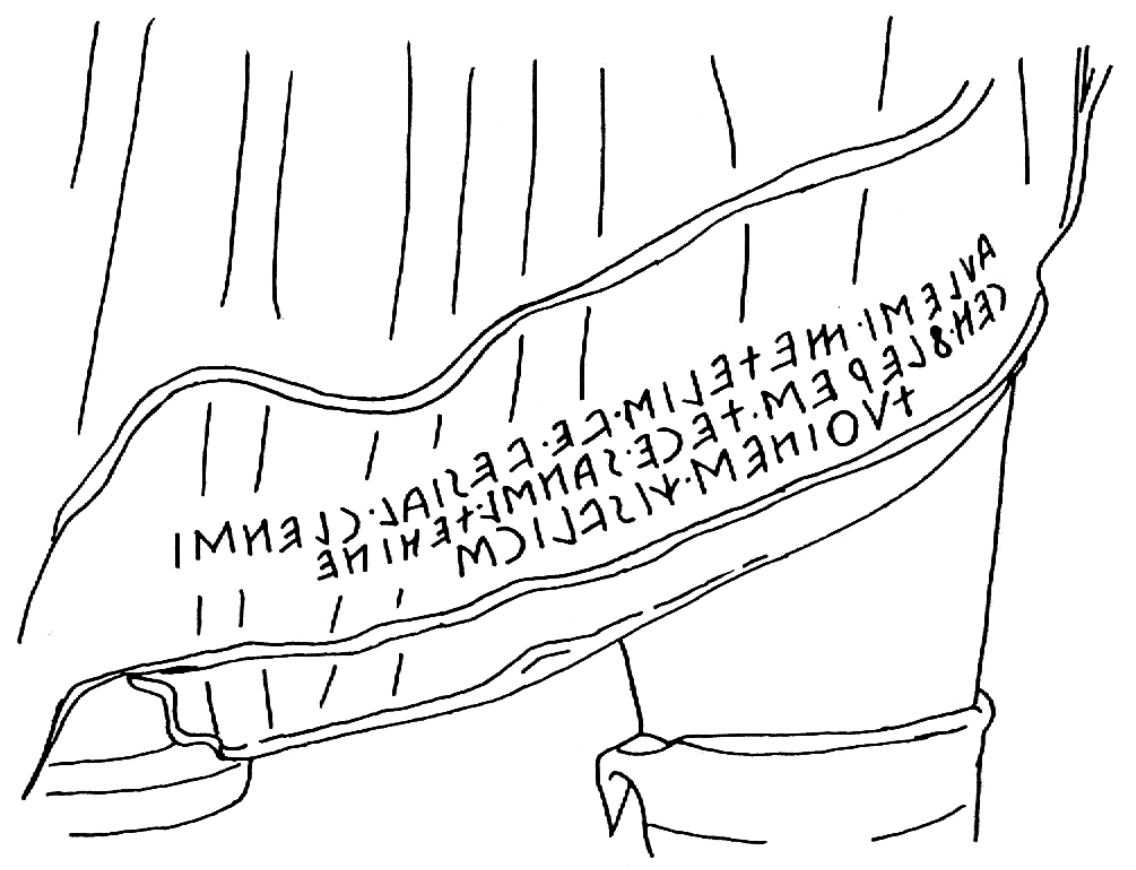

Fig. 3. Tuoro, Lago Trasimeno. Statua di bronzo, cd. "Arringatore" (prima metà del II sec. a.C.)

${ }^{1}$ auleśi . meteliś . ve(luś) . vesial . clenśi ${ }^{2}$ cen . flereś . tece . sanśl . tenine

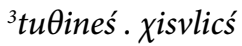

Bibl.: Colonna 1991, 102-106; Maras 2009d, 259, Co do.6; ET Pe 3.3.

\subsection{Scrittura falisca}

Scrittura alfabetica derivante da un precoce adattamento del modello etrusco. ${ }^{30}$ Le più antiche attestazioni risalgono già al VII secolo a.C. e comprendono anche testi di notevole lunghezza, come ad esempio la cosiddetta 'olla di Cerere' conservata al Museo di Villa Giulia.

Nella fase arcaica, la scrittura falisca era sostanzialmente identica al modello standard dell'Etruria meridionale, con particolare riguardo a Veio, salvo una diversa selezione dei segni d'uso che includeva le occlusive sonore $/ \mathrm{g} / \mathrm{e}$ $/ d /($ ma non $/ b /)$ e la vocale /o/, ma escludeva le occlusive aspirate, il digamma e utilizzava il solo sigma per la sibilante. Caratteristico della scrittura falisca e della vicina scrittura capenate fu invece il segno a freccia utilizzato per marcare la spirante labiodentale /f/ sin dal VII secolo a.C.

30 Bibliografia di riferimento: Giacomelli 1963; 2006; Bakkum 2009; Mancini 2011;

Leszkiewicz 2017; Morandi 2017, 83-94; Rigobianco 2019; Biella 2020. 
L'area di distribuzione dei documenti epigrafici falisci è piuttosto limitata e si dispone attorno al centro principale dell'antica Falerii (od. Civita Castellana) con i centri minori di Corchiano, Fabrica di Roma, Vignanello, Gallese, Carbognano e, solo nella fase più recente, S. Maria di Falleri, sede della Falerii di età romana.

La scrittura falisca rimase relativamente stabile nel corso dei secoli e la distinzione tra una grafia arcaica e una recente riposa soprattutto su aspetti formali, in parte dipendenti dall'influenza dei vicini centri etruschi e del latino.

\subsubsection{Tavola dei caratteri}

1. Modelo alfabetico d'uso falisco arcaico (VII-Vsec. a.C.)
A CDE ID
I KLN ${ }^{N}$
$o p$
$P P \leqslant T V$
$\uparrow \phi \xi$
$\uparrow$

2. Modelo alfabetico d'uso falisco recente (IV-II sec. a.C.)
ACDE Y
$R<D E$

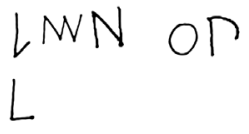
$R \xi+V$
$R 2 Y$

Trascrizione:

a $c d e \quad z \quad h \quad i k r \begin{array}{llllllllllll} & m & n & o & p & q & r & s & t & u & & f\end{array}$

Val. fonetico:

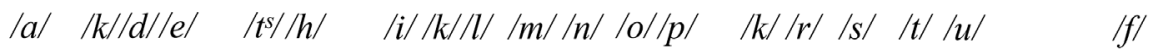

\subsubsection{Esempi illustrativi}

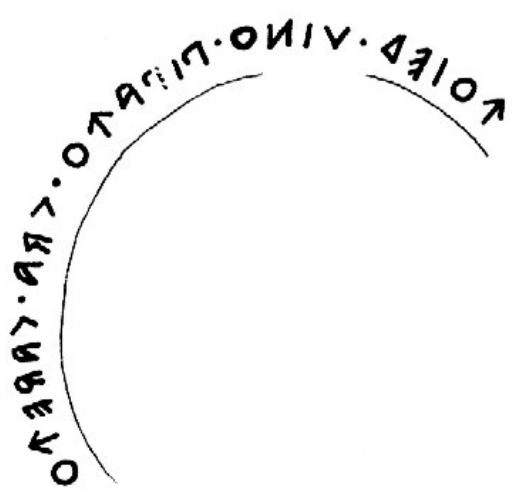

Fig. 4. Civita Castellana. Kylix falisca a figure rosse. (seconda metà del IV sec. a.C.)

foied . uino . pipafo . cra . carefo

Bibl.: Giacomelli 1963, 49-50, n.

5; Bakkum 2009, 434-435, n. 59;

Morandi 2017, 88-89, n. 79. 


\subsection{Scrittura latina}

Scrittura alfabetica derivante da un adattamento del modello etrusco, ancora completo di tutti i segni ereditati dalla serie euboico-calcidese, ma già dotato di proprie norme grafiche, che hanno in parte condizionato la nuova scrittura. ${ }^{31}$ La fonetica del latino aveva bisogno dei segni per marcare le occlusive sonore e la vocale $/ o /$, ma non le occlusive aspirate; inoltre, sin dalle più antiche attestazioni, spesso la semivocale $/ u /$ non viene distinta dalla vocale $/ u /$ ed entrambe sono marcate dalla lettera ypsilon, relegando il digamma a un uso saltuario.

Le norme grafiche etrusco-meridionali trasmesse al latino includevano l'uso del digramma $v h$ per segnare la spirante labiodentale /f/ e l'ortografia delle velari $(k a \sim c e-c i \sim q o-q u)$. Quest'ultima norma provocò una difficoltà d'uso del gamma, destinato a marcare l'occlusiva velare sia sorda che sonora.

$\mathrm{Al}$ principio del VI secolo è in atto un fenomeno di semplificazione dei digrammi, per cui $v h$ si riduce al semplice digamma per il suono / $f$ / (generando così di fatto la lettera F) e $q u$ si riduce alla sola $\mathrm{Q}$ per il continatore della labiovelare sorda. Nello stesso periodo, il segno a croce viene reintrodotto nell'uso per la sibilante composita / $k s /$; la sequenza alfabetica è pertanto chiusa dalla $\mathrm{X}$, come registra il più antico alfabetario latino, ritrovato a Lanuvio $\mathrm{e}$ databile alla fine del VI secolo a.C.

Da questo momento in poi, la serie alfabetica latina è piuttosto stabile e consta di 21 caratteri, includendo $\mathrm{Z}$ al settimo posto e $\mathrm{K}$ al decimo, così come si osserva ancora nellalfabetario di Alsium, dipinto su un piattello Genucilia del principio del III secolo a.C.: ABCDEFZHIKLMNOPQRSTVX. ${ }^{32}$

Per quanto riguarda le velari, tra VI e V secolo si registrano diverse scelte, presumibilmente relative ad altrettanti distretti epigrafici, dei quali solo quello di Roma è ben noto, dove l'occlusiva velare sorda e sonora è marcata dalla sola C. Nel Lazio meridionale, invece, sembra che la preferenza sia stata data soprattutto al $\mathrm{K}$ e sono note alcune varianti grafiche mai attestate a Roma, come le lettere capovolte $(\mathrm{M}, \mathrm{N}, \mathrm{V})$ e la $\mathrm{S}$ a quattro o più tratti.

È significativo che la scrittura latina meridionale sia stata condivisa anche dall'ernica Anagni, come dimostra un unico documento epigrafico,

31 Bibliografia di riferimento: Bernardi Perini 1983; Rocca 1997; Urbanovà 1999; Attenni e Maras 2004; Mancini 2004; 2011; Fortini 2005; Maras 2005; 2009a; 2009b; 2009c; Mangani 2015; Muscariello 2015; Morandi 2017, 67-82. 
proveniente dal deposito votivo di Santa Cecilia e datato alla fine del VI secolo a.C. (ST He 2). Lunica differenza che si riscontra è il segno della $\mathrm{V}$ tagliata, che forse serviva a marcare una vocale intermedia $\langle\dot{u}\rangle$ (a meno che non sia dovuto a un mero errore dello scriba). ${ }^{33}$

\subsubsection{Tavola dei caratteri}

1. Modelo alfabetico d'uso latino arcaico (VI-V sec. a.C.)

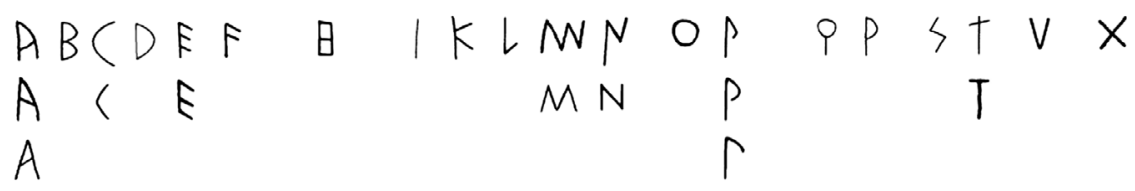

2. Varianti grafiche attestate solo a Roma
A
$L N$
$\uparrow$
P s
$\wedge$

3. Varianti grafiche attestate fuori Roma

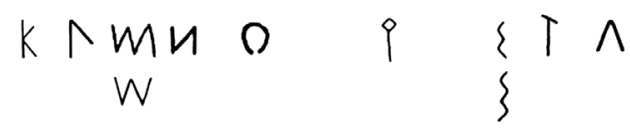

Trascrizione:

$\begin{array}{lllllllllllllllll}A & B C D E F / V & H & I & K L & M & N & O & P & Q & R & S & T & U & X\end{array}$

Val. fonetico:

$|a / / b / / k / / d / / e / f f-u / \quad / h / \quad / i / / k / / l / / m / / n / / o / / p / \quad / k / / r / / s /| t / / u / \mid k s /$ 


\subsubsection{Esempi illustrativi}
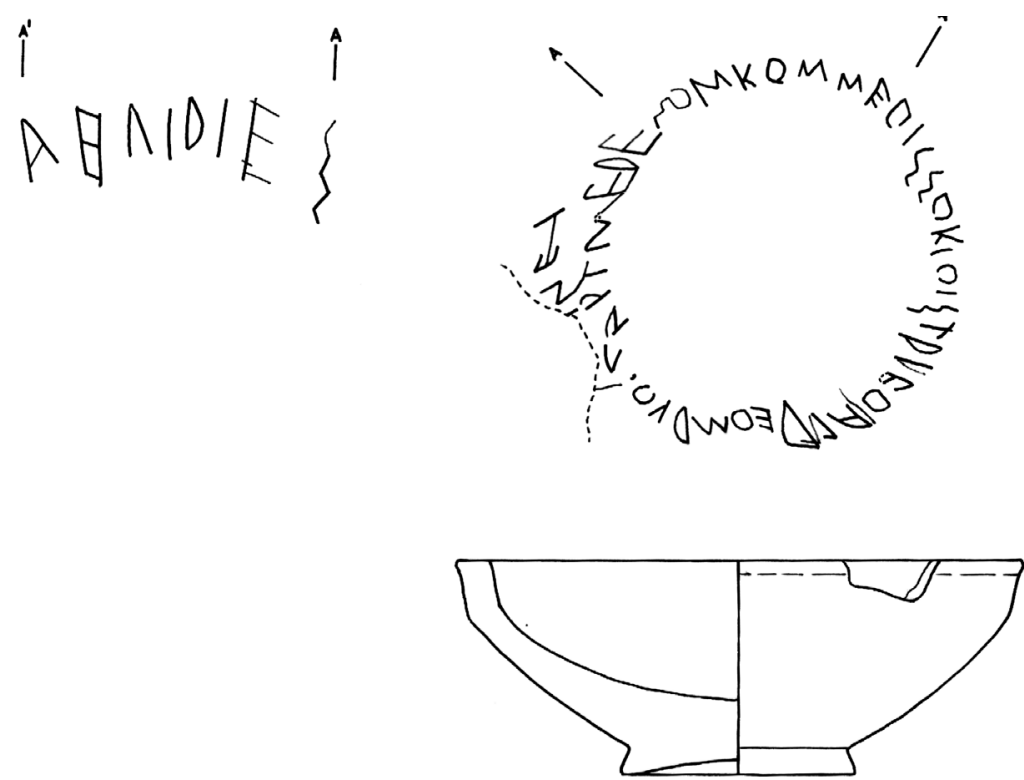

Fig. 5. Minturno, santuario alla foce del Garigliano. Coppa d'impasto (fine del VI sec. a.C.) ${ }^{a}$ ahuidies ${ }^{b, 1}$ esom kom meois sokiois trivoial deom duo . ${ }^{2} \times[-(-)] \times$ nei ${ }^{3}$ pari med Bibl.: Maras 2005; De Simone 2006; Morandi 2009; Mancini 2011, 251-256.

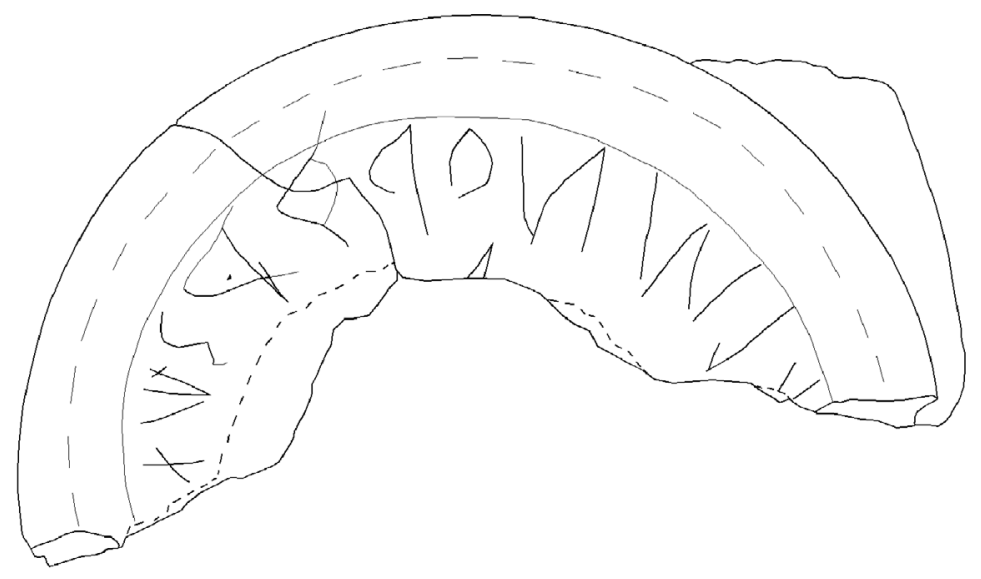

Fig. 6. Lanuvio, santuario di Iuno Sospita. Coppa frammentaria di bucchero grigio (ultimo quarto del VI sec. a.C.)

[---]klm nopqrstux

Bibl.: Attenni e Maras 2004, 61-78; Maras 2009b, 105-107. 


\subsection{Scrittura golasecchiana (leponzia)}

Scrittura alfabetica derivata dal modello etrusco settentrionale, per il tramite dei rapporti di dono e ospitalità tra le aristocrazie. ${ }^{34}$ Le più antiche attestazioni risalgono alla fine del VII secolo a.C. e documentano una fase, durata almeno fino alla prima metà del secolo seguente, in cui scribi etruschi erano all'opera come maestri per la trasmissione e l'adattamento della scrittura alla lingua locale. Un ruolo chiave per il primo accoglimento e rielaborazione della scrittura ha avuto il nucleo proto-urbano di Sesto Calende - Golasecca - Castelletto Ticino.

Significativa in questa prima fase è la decisione di utilizzare i segni che nel modello etrusco marcavano le coppie oppostive delle occlusive sorde e aspirate per le corrispettive coppie sorde e sonore: tale fenomeno, forse dovuto a un fattore di economia e alla preferenza per i segni d'uso piuttosto che per quelli del corpus dottrinale ${ }^{35}$ rende necessario ricorrere a una doppia trascrizione delle iscrizioni: 'diplomatica' per il valore originale dei segni vs. 'interpretativa' per il loro valore fonetico attuale (p. es. dipl. $t=$ int. $d=$ fon. $/ d /$; dipl. $\varphi$ $=$ int. $b=$ fon. $/ b /$; dipl. $\chi=$ int. $k=$ fon. $/ k /$ ). Nel tempo, oscillazioni grafiche e variazioni del sistema scrittorio rendono a volte difficile essere sicuri della corrispondenza fonologica di alcuni segni.

A partire dalla seconda metà del VI e nel V secolo a.C., si diffonde un tipo di scrittura lapidaria monumentale (cd. II fase), in cui le lettere sono incorniciate da binari incisi, che spesso compaiono anche sui testi vascolari e dipendono da una tradizione scribale. In questa fase è particolarmente attiva una scuola scrittoria nel nucleo di Como-Prestino. A questa tradizione si rifa anche la moda grafica peculiare che caratterizza le stele iscritte del Canton Ticino tra fine $\mathrm{V}$ e prima metà IV secolo a.C. (cd. III fase). ${ }^{36}$

Da segnalare gli sviluppi della forma dell'alpha a bandiera (in parallelo alla caduta in disuso del digamma) e del tsade a farfalla, tipici della scrittura golasecchiana.

34 Bibliografia di riferimento: Lejeune 1971; De Marinis e Motta 1990-1991; Prosdocimi 1991; Solinas 1995; 2002; Motta 2000; Rocca 2000, 196-198; Sassatelli 2000; Verger 2001; Morandi 2004; Rubat Borel 2005; Gambari 2012; Maras 2014a; 2014b; Morandi 2017, 403-437.

35 Per l'uso del termine, si veda Prosdocimi 1990, 151-159. 
Infine, in epoca Lateniana (cd. IV fase, IV-I secolo a.C.), la scrittura celtica d'Italia assume una forma standard semplificata, caratterizzata dall'abbandono di alcuni segni (theta, digamma, qoppa, phi), che lavrebbe accompagnata fino alla romanizzazione in attestazioni che eccedono la regione golasecchiana originale e si estendono ampiamente nella pianura padana e lungo la penisola italiana. ${ }^{37}$

\subsubsection{Tavola dei caratteri}

1. Modello alfabetico d'uso di I fase (fine VII - inizio VI secolo a.C.)
A $\pi 1$
?
$\odot \mid \begin{array}{r}X \\ ?\end{array}$
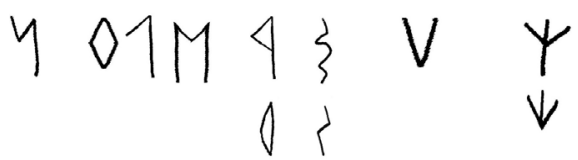

2. Modello alfabetico d'uso di II fase (VI - V secolo a.C.)
A
$\exists 7 I$
$\odot \mid>1 \sqrt{ } y$
$01 \AA P O$
W
(1) $\downarrow$
$\neq$

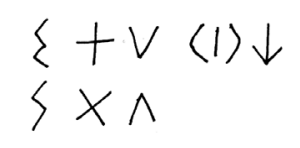

3. Modello alfabetico d'uso di III fase (fine V - prima metà IV secolo a.C.)

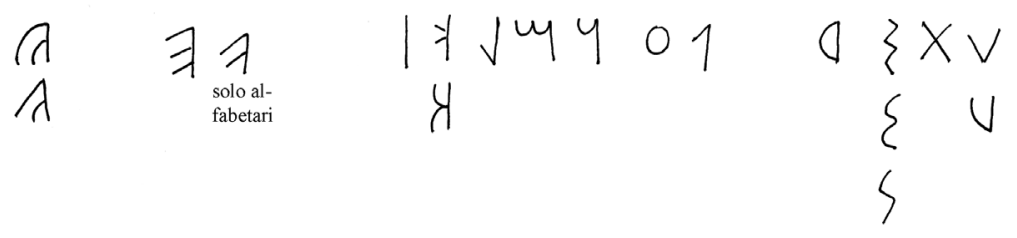

4. Modello alfabetico d'uso di IV fase (fine IV - I secolo a.C.)

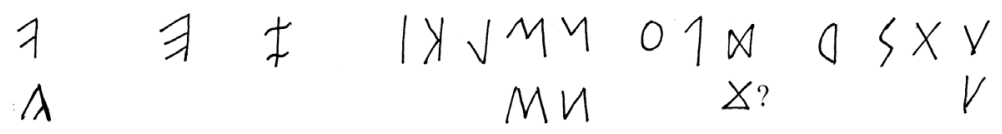

Trascrizione:

$a$ $\begin{array}{lllllllllllllllllll}e & v & z & \theta & i & k & l & m & n & o & p & s & q & r & s & t & u & \phi & \chi\end{array}$

Val. fonetico:

lal

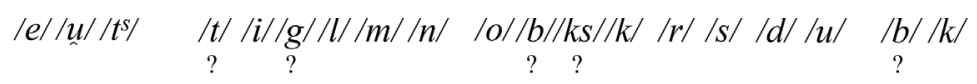

37 Solinas 2002. 


\subsubsection{Esempi illustrativi}
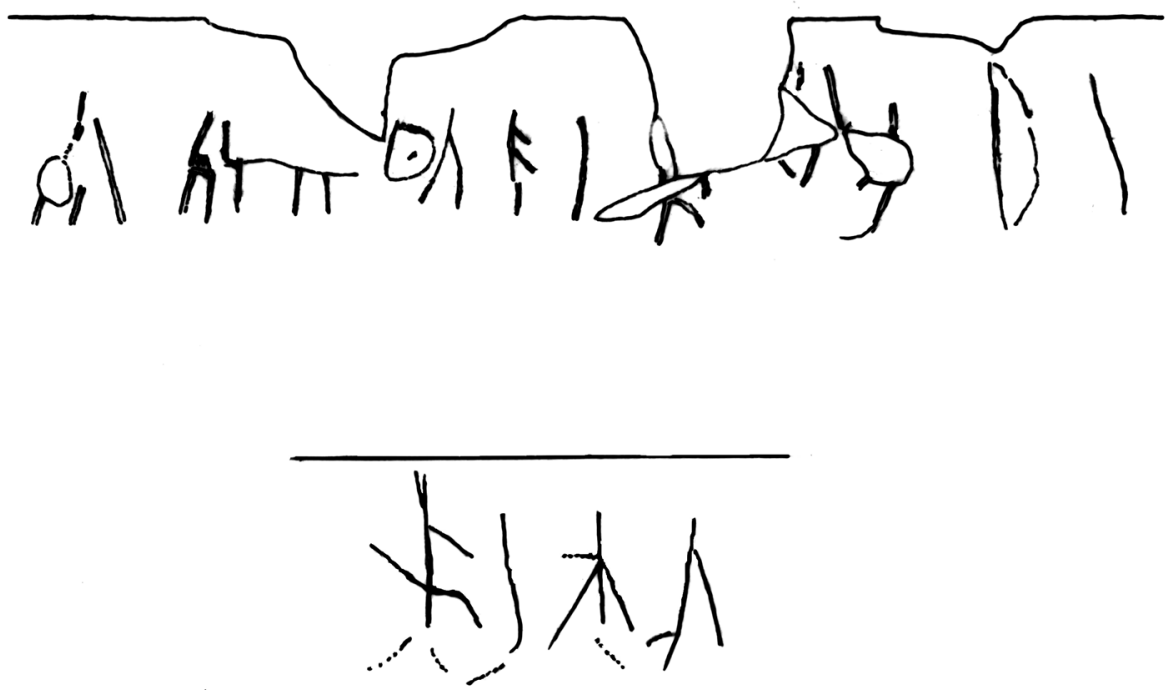

Fig. 7. Sesto Calende, tomba 12 della necropoli di via Sculati. Boccale d'impasto (primo quarto del VI sec. a.C.)

${ }^{a} u \underline{e ̣} \theta u$ vik $\times o ̣ \times r i{ }^{b} v i \chi u$ (in etrusco)

Bibl.: R.C. de Marinis, in De Marinis et al. 2009, 423-425; Maras 2014c, 104-106.

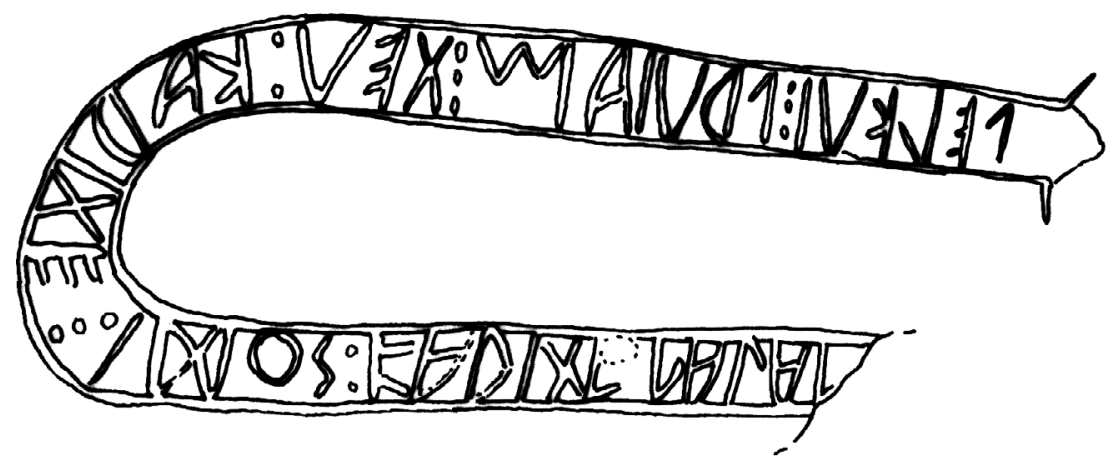

Fig. 8. Vergiate (VA). Stele di pietra (fine del VI-inizio del V sec. a.C.) pelkui : pruiam : teu : karite : iśos [:] karite : palam

Bibl.: Lejeune 1971, 88-96; Morandi 2004, 594-596, n. 106; Maras 2014a, 81, n. 7. 


\subsection{Scrittura veneta}

Scrittura alfabetica derivata dal modello etrusco in due fasi distinte, probabilmente connesse con diversi canali di trasmissione. ${ }^{38}$

Un piccolo numero di iscrizioni databili ancora nel pieno VI secolo a.C. presentano un modello alfabetico etrusco settentrionale, affine a quello chiusino, con delle norme di adattamento fonologico simili a quelle in uso nella coeva scrittura golasecchiana. In assenza dei segni per le occlusive sonore, le coppie oppositive sono state formate con i segni per le aspirate, ma in modo asimmetrico: $t=/ d / \sim \theta=/ t /$, ma $k=/ k / \sim \chi=/ g /$. Il theta è del tipo a croce di Sant'Andrea e due diverse sibilanti sono espresse rispettivamente da sigma e tsade.

Un secondo modello alfabetico viene introdotto verso la fine del VI secolo a.C., probabilmente in occasione dell'istituzione di una scuola scrittoria nel santuario della dea Reitia a Este. In questa tradizione, le lettere assumono forme quadrangolari e si prediligono linee spezzate e oblique (particolarmente adatte a tecniche di incisione su metallo e pietra). Sviluppi caratteristici vedono nel tempo l'alpha a bandiera chiusa e poi aperta; lo het a scaletta e poi ad asta verticale con punti laterali; il theta a rombo puntato e poi a croce di Sant'Andrea.

Le occlusive sonore sono espresse dai corrispondenti segni etruschi per le aspirate nel caso delle labiali e delle velari, secondo lo schema $p=\mid p / \sim \varphi=/ b /$ vs. $k=/ k / \sim \chi=/ g /$. Viceversa, per le dentali resta l'equivalenza $\theta=/ t /$, mentre la sonora viene espressa dapprima dal tau (sia a traversa verticale che a croce di Sant'Andrea) e poi da zeta (nella forma obliqua con due traverse secanti). Come per la scrittura celtica, anche questo complesso sistema richiede una doppia trascrizione, diplomatica e interpretativa, delle iscrizioni.

Lo sviluppo del sistema scrittorio è stato attribuito ipoteticamente a diverse fasi di insegnamento della scrittura nella scuola santuariale di Este, accolte più o meno estesamente dai centri vicini di Padova, Altino e del vicentino.

Nell'insieme, la scrittura veneta mantiene le proprie caratteristiche arcaizzanti e il proprio peculiare aspetto formale fino alla diffusione della scrittura latina e oltre. 


\subsubsection{Tavola dei caratteri}

1. Modello alfabetico d'uso di I fase (VI secolo a.C.)
A
11
$\theta \times|\geqslant| y \eta 0$
M $9<T$
$Y$

2. Modello alfabetico d'uso di II fase (fine VI-I sec. a.C.)
$a$
习习x
$\triangleleft<\uparrow \vee \bowtie \vee$
$3 x$
$\lambda$

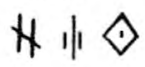
$M H$

Trascrizione:

$a$

$$
\begin{array}{lllllllllllllllllllllllll}
e & v & z & h & \theta & i & k & l & m & n & o & p & s & & r & s & t & u & \phi & \chi
\end{array}
$$

Val. fonetico:

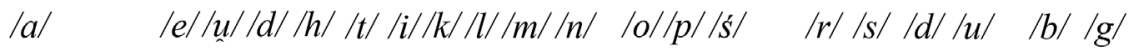

\subsubsection{Esempi illustrativi}

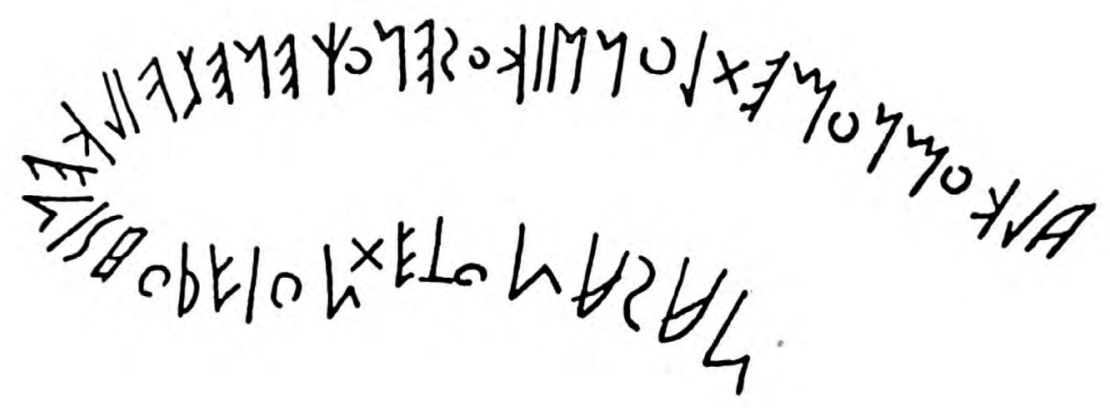

Fig. 9. Lozzo Atestino (PD). Kantharos di bronzo (prima metà del VI sec. a.C.)

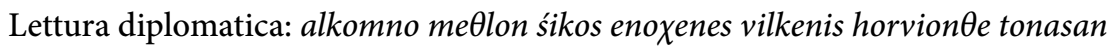
Lettura interpretativa: alkomno metlon śikos enogenes vilkenis horvionte donasan Bibl.: Lejeune 1974, 243-246, n. 123; D. Locatelli e A. Marinetti, in Marinetti 2002, 157-158, n. 1. 


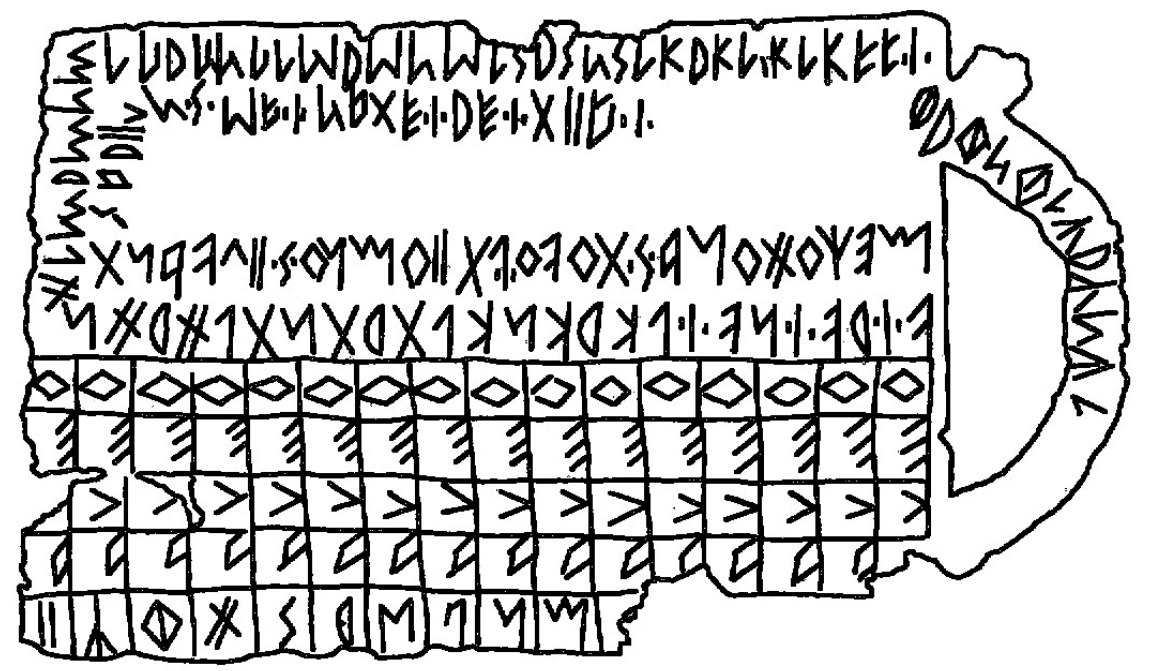

Fig. 10. Este (PD), santuario di Reitia, stipe di Baratella. Tavoletta-alfabetario di bronzo (V-IV sec. a.C.)

Lettura diplomatica: mexo zona.s.to vo.l.tiiomno.s. iiuvant/s ariiu/n.s. śa.i.nate.i. re.i.tiia.i.

Lettura interpretativa: mego donasto voltiomnos iuvants ariuns śainatei reitiai Bibl.: Pellegrini e Prosdocimi 1967, I, 109-111, Es 25; Lejeune 1974, 197-198, n. 10.

\subsection{Scrittura retica}

Scrittura alfabetica derivata dal modello etrusco arcaico a partire almeno dalla metà del VI secolo a.C., diffusa lungo la valle dell'Adige, attorno ai due poli fondamentali di Sanzeno in Alto Adige e Magré nel vicentino. ${ }^{39}$ Ladattamento fonologico della scrittura è stato reso più facile dalla parentela linguistica tra l'etrusco e il retico, ma non è stato comunque privo di conseguenze. Il modello di partenza era di tipo settentrionale, con il solo kappa per la velare sorda e le due sibilanti sigma e tsade; il theta non viene utilizzato, ma nell'uso compare invece un diverso segno che evidentemente esprime un altro tipo di marca per la dentale (sulla cui natura non si è ancora raggiunta una posizione condivisa), trascritta per convenzione come $\left\langle t^{\prime}\right\rangle$. Per esprimere tale suono vengono 
utilizzati due diversi segni, caratteristici di modelli alfabetici alternativi: il beta a tre occhielli a Magré e la freccia verso l'alto a Sanzeno.

Nell'insieme i segni presentano una forma angolosa (anche in conseguenza dei supporti scrittori, di regola soprattutto incisioni su osso e bronzo) e si riscontra una tendenza a ruotare e capovolgere le lettere.

\subsubsection{Tavola dei caratteri}

Modello alfabetico d'uso di IV (VI - I secolo a.C.)

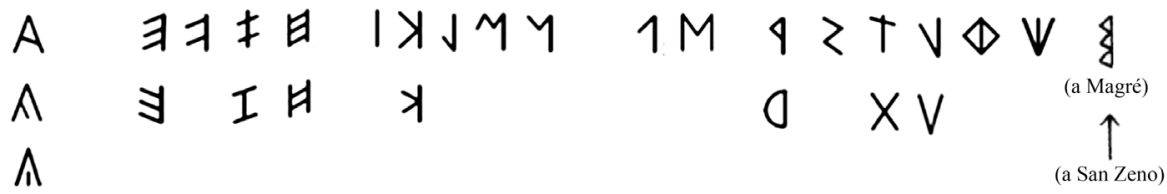

Trascrizione:

$\begin{array}{lllllllllllllllllll}a & e & v & z & h & i & k & l & m & n & p & s & r & s & t & u & \phi & \chi & t^{\prime}\end{array}$

Val. fonetico:

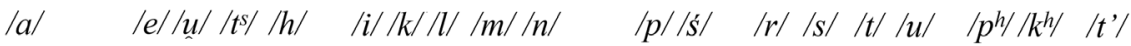

\subsubsection{Esempi illustrativi}
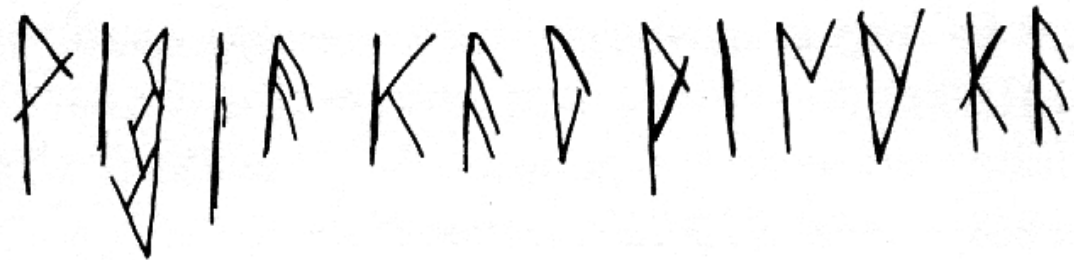

Fig. 11. Magrè (VI). Corno di cervo votivo (III sec. a.C.)

rit'ie ker rinake

Bibl.: Schumacher 1992, 163, n. 10; Morandi 1999, 76-78, n. 37; Marchesini 2015. 


\subsection{Scrittura camuna}

Scrittura alfabetica derivata dal modello etrusco, ma con connessioni ad altre tradizioni alfabetiche ancora da approfondire. ${ }^{40}$ La scrittura camuna è documentata esclusivamente da iscrizioni rupestri distribuite lungo una serie di siti montani della Val Camonica e prive pertanto di contesti archeologici o dati derivanti dall'analisi del supporto.

Sembra verosimile che il contesto originario della trasmissione della scrittura sia stato simile a quello delle altre grafie dell'Italia settentrionale derivate dal modello etrusco nell'arco del VI secolo a.C.; ciò nonostante, va considerato che a differenza del celtico, del veneto e del retico, la serie alfabetica camuna nota dagli alfabetari rupestri di Piancogno ${ }^{41}$ conserva anche i segni delle occlusive sonore, sebbene sembra che questi non siano stati utilizzati nella scrittura.

Inoltre, alcune scelte grafiche e la forma peculiare di alcuni segni suggeriscono una più lunga gestazione e la presenza di influenze esterne, forse di ambito italico meridionale. A quest'ambito rimanda infatti la forma del pi, che richiama da vicino il digamma uncinato enotrio-ausone e paleo-sabellico, nonché la sibilante ad alberello, identica al segno corrispondente ausone (v. oltre).

\subsubsection{Tavola dei caratteri}

Modello alfabetico d'uso

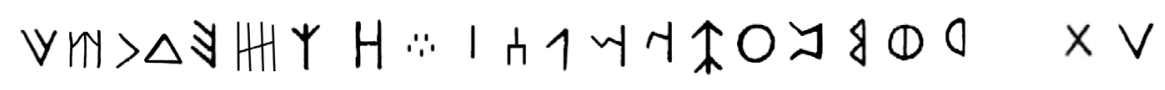

Trascrizione:

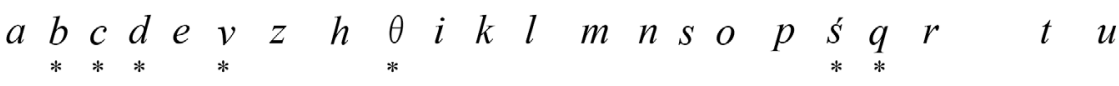

(* solo negli alfabetari)

40 Bibliografia di riferimento: Mancini 1980; Tibiletti Bruno 1990; Marchesini 2011; Morandi 2017, 439-456.

41 Tibiletti Bruno 1990, 67-76; Morandi 2017, 450, n. 628. 


\subsubsection{Esempi illustrativi}

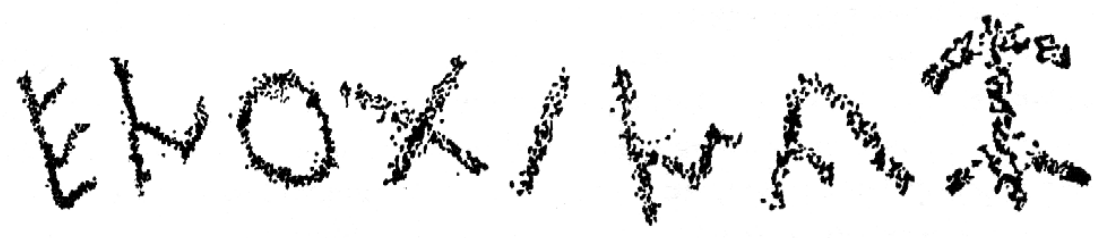

Fig. 12. Cimbergo (BS). Roccia con iscrizioni scolpite (cronologia incerta) enotinas

Bibl.: Mancini 1980, 90; Morandi 2017, 447-448, n. 622.

\subsection{Scritture paleo-italiche}

Tradizione grafica documentata da un piccolo gruppo di iscrizioni arcaiche dellarea tiberina presumibilmente derivanti dal modello etrusco, ma con esiti alquanto vari e poco sistematici. ${ }^{42}$

Sotto l'etichetta di scritture paleo-italiche si raccolgono i seguenti documenti epigrafici in lingua italica: a) il biconico di Uppsala, attribuito all'Umbria tiberina, terzo quarto del VII secolo a.C. (ST Um 41); b) l'olletta di Colle del Giglio, ultimo quarto del VII secolo a.C. (ST Um 3); c) la fiaschetta di Poggio Gaiella (Chiusi), fine del VII secolo a.C. (ST Um 40); d) la fiaschetta di Poggio Sommavilla, inizi del VI secolo a.C. (ST Um 2); e) il cratere del Ferrone (Tolfa), prima metà del VI secolo a.C. (ST Um 4); f) l'accettina di Satricum, prima metà del V secolo a.C. (ST VM 1).

Sebbene non riducibili a un'unico modello alfabetico, le iscrizioni qui menzionate possono essere raggruppate in base ad alcuni elementi grafici, che comparvero in questo gruppo per la prima volta e successivamente si diffusero ampiamente nelle scritture dell'Italia centro-meridionale.

La derivazione dal modello etrusco è postulabile sulla base dell'uso del gamma per la velare sorda nell'iscrizione del Ferrone (e); ma va rilevato l'uso dell'intero modello alfabetico euboico-calcidese, comprendente anche i segni in disuso nell'etrusco, come beta, delta, omicron e il samekh a cancelletto. Quest'ultimo segno è utilizzato per la vocale intermedia $<i<$ e a volte presenta 
interferenze con altri segni che ne diventano varianti, come circolo crociato (originariamente un theta in a) e il segno a scaletta (originariamente un het in d). Da segnalare anche l'uso del $c h i$ a tridente per la vocale $\langle\dot{u}\rangle$, del segno a $<8>$ (o doppio circolo) per la spirante labiodentale /f/ (b, d, f), in alternativa al segno a freccia (e). Infine, è significativa la riduzione a un semplice punto del cerchio della /o/ (a, f) e di quelli del segno a $<8>$ (f).

Nell'insieme, si direbbe che queste scritture locali (quasi 'familiari' tra le élites italiche) siano state un vero laboratorio di innovazioni per la costruzione di una tradizione grafica italica indipendente da quella greco-etrusca tra VII e V secolo a.C.

\subsubsection{Tavola dei caratteri}

1. Biconico di Uppsala (terzo quarto del VII secolo a.C.)

8

$\oplus \cdot$

7

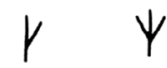

2. Olletta di Colle del Giglio (ultimo quarto del VII secolo a.C.)
A
$\triangle E$
D $1 \mathrm{~K}$
田 0
$P \leqslant T Y$
$:$

3. Fiasca di Poggio Gaiella (fine del VII secolo a.C.)
b
太
$1 M N \otimes$
$\{广$

4. Fiaschetta di Poggio Sommavilla (inizio del VI secolo a.C.)
夙
(D) $\{$ [
B. 6 迹
(B) @
P $\ll$
0

5. Cratere del Ferrone (prima metà del VI secolo a.C.)
$A \cap \Pi$

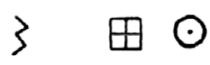
$\xi T \cap$
$\uparrow$

6. Accettina di Satricum (prima metà del V secolo a.C.)

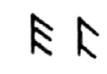

I K $\hat{?}$

Trascrizione:

$a b c d e v z \quad h \quad i k l \quad m \quad n \quad i \quad o p \quad r \quad r s \quad t \quad u \quad u f$

Val. fonetico:

$\mid \mathrm{a} / / \mathrm{h} / / \mathrm{k} / \mathrm{d} / / \mathrm{e} / \mathrm{lol} / \mathrm{ts} / \mathrm{h} /$
$?$ 


\subsubsection{Esempi illustrativi}

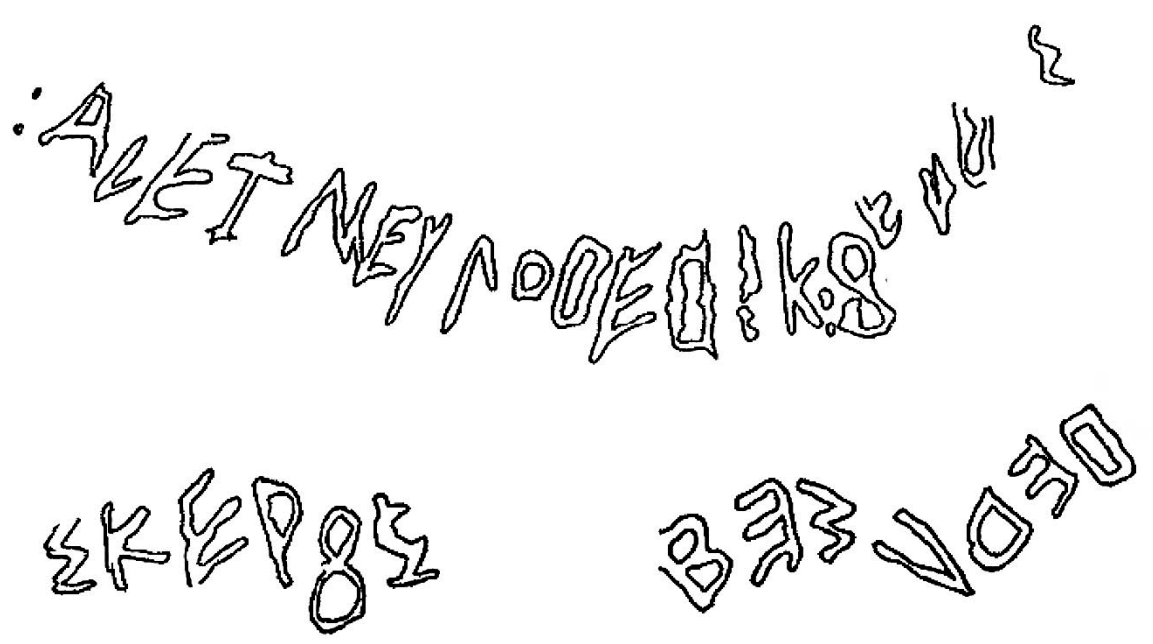

Fig. 13. Poggio Sommavilla. Fiaschetta miniaturistica di impasto (fine del VII sec. a.C.) a: alez me upo hehik(e) ferhs ${ }^{b}$ skerfs ${ }^{c}$ heduseí (ovvero heruseí)

Bibl.: Rix 1996; Rocca 2000, 181, figg. 2-3; ST Um 2; Agostiniani et alii 2011, 13-14, n. 4; Maras 2012b.

\subsection{Scrittura paleo-sabellica}

Scrittura alfabetica (anche detta sud-picena) derivante dal modello etrusco per il tramite della tradizione paleo-italica alto-arcaica e documentata da un gruppo di ca. 25 iscrizioni, soprattutto su stele funerarie, databili tra il secondo quarto del VI secolo a.C. (il 'Guerriero di Capestrano', ST Sp AQ 2) e il IV secolo a.C. (l'elmo di Bologna, ST BO 1). ${ }^{43}$

Il modello alfabetico riprende e porta a compimento alcune delle innovazioni viste in nuce nelle scritture paleo-italiche, come l'uso del samekh per la $<i>$ (ridotto a un segno a scaletta o a rettangolo vuoto), del tridente per la $<\dot{u}>$ (con il tratto centrale staccato) e la riduzione a semplici punti dei circoli della $<0>$ e del segno a $<8>$. Si segnala inoltre nel corso del tempo l'introduzione di nuovi segni (come l'asterisco) e varianti (come il digamma apicato e il tau con traversa ridotta a un punto). 
La lingua attestata dalle iscrizioni è italica, affine al paleo-umbro, ${ }^{44} \mathrm{e} \mathrm{sem-}$ bra mantenere le proprie caratteristiche costantemente dall'età arcaica fino alle attestazioni più recenti.

\subsubsection{Tavola dei caratteri}

Modello alfabetico d'uso

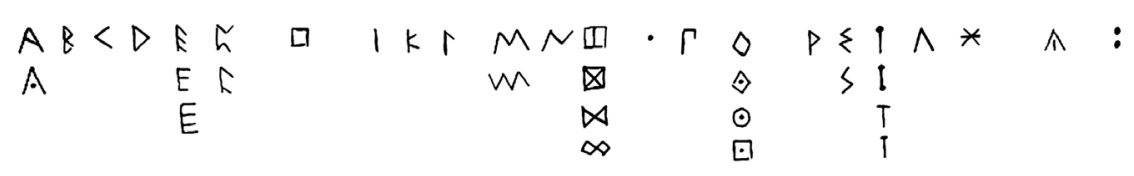

Trascrizione:

$\begin{array}{lllllllllllllllllllll}a b & c & d & e & v & h & i k & l & m & n & i & o & p & q & r & s & t & u & \text { ? } & u & f\end{array}$

Val. fonetico:

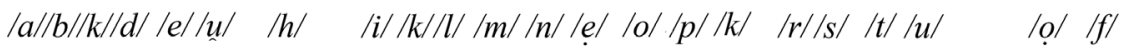

\subsubsection{Esempi illustrativi}

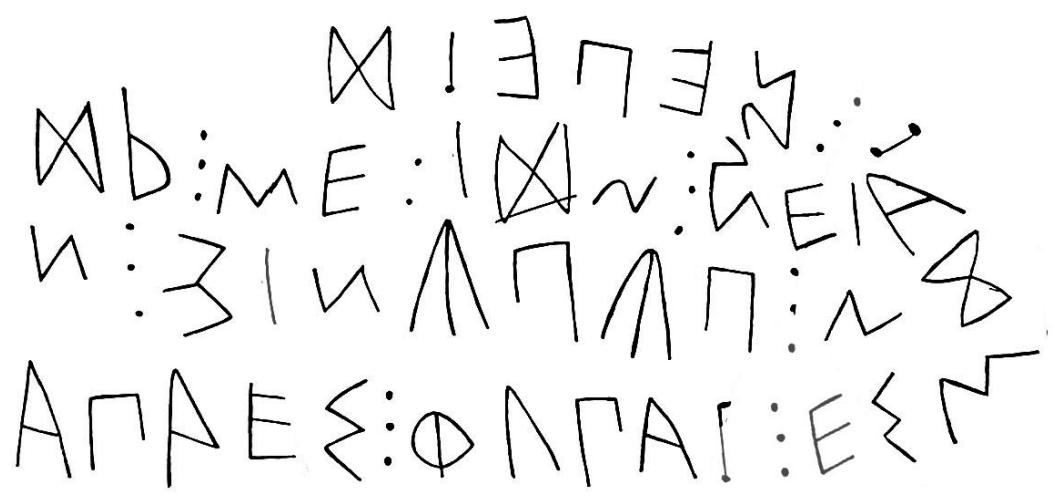

Fig. 14. Loro Piceno (MC). Stele in arenaria (VI sec. a.C.) apaes : qupat [: e]sm/ín : púpún[i]s : n/ír : mefín : veia/t : vepetí Bibl: Marinetti 1985, 106-107, e 161-164, MC.1; ST Sp MC 1; Morandi 2017, 115116, n. 105. 


\subsection{Scrittura ausone (alfabeto nocerino)}

Scrittura alfabetica derivata dal modello greco euboico di Cuma (o, secondo alcuni, da un modello greco ionico importato da Velia) e diffusa in area sorrentina tra VI e V secolo a.C. ${ }^{45}$ Le tre iscrizioni maggiori appartenenti a questa tradizione vengono da Nocera, Vico Equense e Sorrento ${ }^{46} \mathrm{e}$ si riferiscono a una lingua italica arcaica, di regola associata alle attestazioni pre-sannitiche della Campania e del Lazio.

Nella pesante rielaborazione formale dei segni e in alcune scelte grafiche peculiari, sembra evidente la volontà di marcare fortemente la differenza tra questa scrittura e quelle greca ed etrusca, diffuse nello stesso periodo in Campania. In particolare, si sottolinea la scelta singolare di usare una variante ad alberello del samekh (con due o tre ordini di traverse) per la sibilante $/ \mathrm{s} / \mathrm{e}$ di rendere con un digamma apicato la spirante labiodentale /f/. Quest'ultima caratteristica è condivisa con la scrittura enotria documentata dal Cippo del Tortora e non può essere considerata del tutto indipendente dalla comparsa del medesimo segno nella scrittura paleo-sabellica (per la $/ u /$ semivocalica) e forse, a grande distanza, nella scrittura camuna (per la $/ p /$ ).

\subsubsection{Tavola dei caratteri}

Modello alfabetico d'uso

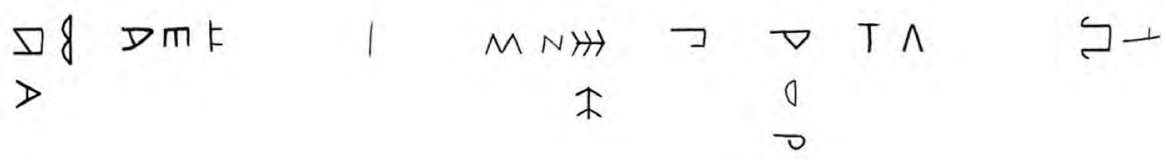

Trascrizione:

$\begin{array}{lllllllll}a b & d & e & & & \end{array}$

Val. fonetico:

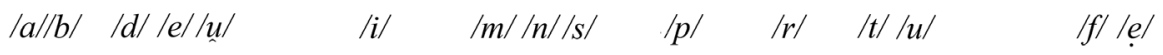

45 Bibliografia di riferimento: Colonna 1994; Russo 2005, 27-81; Agostiniani 2010; Poccetti 2010; Crawford 2011, 16, 851-853 e 909-910; Calderini 2012; Tikkanen 2020, 105.

46 Rispettivamente Russo 2005 e ES Ps 4-5. 


\subsubsection{Esempi illustrativi}
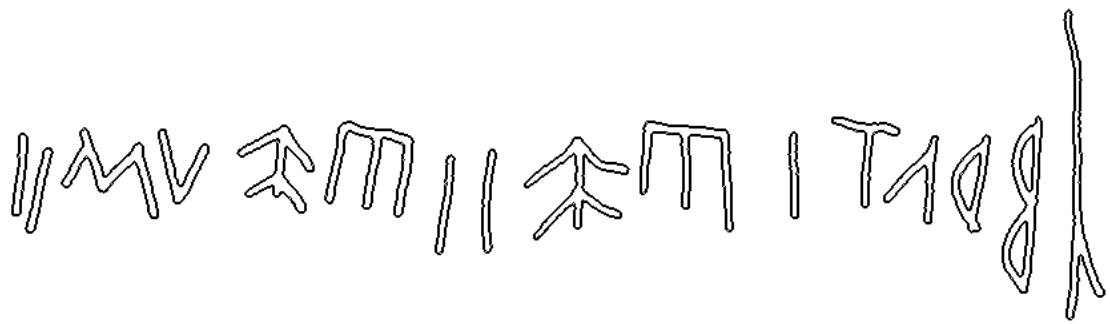

Fig. 15. Nocera. Oinochoe di bucchero (metà del VI sec. a.C.)

| bruties || esum ||

Bibl.: Colonna 1994, 84-86; Russo 2005, 61, NS 2; ST Ps 4.

\subsection{Scrittura osca}

Scrittura alfabetica derivata dal modello etrusco, dopo una lunga gestazione in Campania nel corso del V secolo a.C. (cd. iscrizioni 'pre-sannitiche'), non priva di influenze dall'ambito ausone e paleo-sabellico. ${ }^{47}$ In particolare, dalla scrittura etrusca sembrano essere stati presi l'assenza del segno per la /o/ e l'uso del segno a $<8>$ per la $/ f /$; ciò nonostante, l'alfabeto conserva i segni per le occlusive sonore $/ b-d-g /$.

A partire da una riforma grafica alla fine del IV secolo a.C. le vocali intermedie $<i<$ e $<\dot{u}>$ vengono regolarmente espresse con i segni di iota e ypsilon diacriticati, la cui origine ultima si trova nella più antica scrittura ausone nocerina. L'alfabeto definitivo, con forme squadrate molto regolari e con poche varianti grafiche, arrivò così a contare 21 segni.

Tra tutte le tradizioni scrittorie dell'Italia preromana, va rilevato come quella osca rimase particolarmente stabile nel tempo, diffondendosi ampiamente presso le popolazioni italiche del Sannio e della Campania fino alla romanizzazione e anche oltre, dal momento che i testi più recenti possono essere datati al I secolo d.C. ${ }^{48}$

47 Bibliografia di riferimento: Colonna 1984; Del Tutto Palma 1996; Rocca 2000, 189-192; Untermann 2000; Cooley 2002; Wallace 2004a; 2007; Crawford 2011; Morandi 2017, 187-242; Tikkanen 2020.

48 Benelli 2001; Cooley 2002; vedi anche Farney 2011. 


\subsubsection{Tavola dei caratteri}

Modello alfabetico d'uso
Пઇ>ク习コ I 日
$1 \times \sqrt{ } \mathrm{MH}$
$\Pi \triangleleft \checkmark \uparrow \vee$
$\vee 8-1$

Trascrizione:
$a b g d e v z h$
$\begin{array}{lllll}i & k & l & m & n\end{array}$
$p$
$\begin{array}{llll}r & s & t & u\end{array}$
ú $f \quad i$

Val. fonetico:
la//b//g//d/le//u//ss//h/
li/ $/ k / / l / / m / / n /$
$. p / \quad|r /| s /|t| / u \mid$
lol $|f| l e l \mid$

\subsubsection{Esempi illustrativi}

\section{MV々・২トヨTกTHコロ}

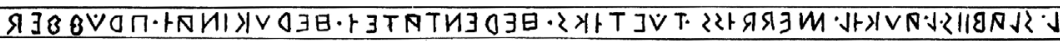

Fig. 16. Ercolano. Altare di marmo (II sec. a.C.)

${ }^{a}$ herentateís . súm ${ }^{b} l$. slabiis . l. aukíl . meddíss . túvtíks . herentateí . herukinaí . prúffed Bibl.: ST Cm 10; Morandi 2017, 209-210, n. 169.

\subsection{Scrittura umbra}

Scrittura alfabetica derivata dal modello etrusco: ${ }^{49}$ si tratta fondamentalmente di un adattamento diretto della scrittura etrusca in uso nell'ambito interno tiberino, con particolare riguardo all'area volsiniese nella fase più antica, ancora nel V secolo a.C., e poi a quello Perugino e Cortonese per le fasi più recenti. Uniche concessioni alla necessità di introdurre segni grafici specifici per marcare suoni della fonologia locale sono il segno a $\mathrm{P}$ con occhiello aperto, presente sin dalla prima documentazione per segnare l'esito (forse monovibrante o fricativo) dellocclusiva dentale sonora antevocalica, trascritto come $<\check{r}>$, e il segno opposto $\mathrm{b}$, che non compare prima del III secolo a.C. e serve a marcare una fricativa palatale, trascritta come $\langle c ̧>$.

49 Bibliografia di riferimento: Prosdocimi 1984; Ancillotti e Cerri 1996; Rocca 1996; Untermann 2000; Wallace 2004a; 2007; Ancillotti e Calderini 2009; Sisani 2009; Agostiniani et alii 2011, 3-7 e 21-89; Crawford 2011; Morandi 2017, 133-186. 
La prossimità e il continuo interscambio con la scrittura etrusca d’oltre-Tevere sono ulteriormente dimostrate dalladozione dello het circolare e del $m y$ a V rovesciata tipici dell'area cortonese nel corso del III-II secolo a.C.

Documento principe della scrittura e lingua umbra sono le Tabulae Iguvinae (ST Um 1), un gruppo di sette tavole di bronzo datate tra la fine del III e gli inizi del I secolo a.C. contenente un intero corpus di prescrizioni rituali e preghiere relative alla città di Gubbio. ${ }^{50}$ Cinque tavole sono iscritte nella grafia umbra, tutte opistografe tranne due, mentre parte della faccia posteriore della quinta e le ultime due (in tutto cinque facciate) sono state iscritte utilizzando lalfabeto latino adattato alla lingua locale, in un momento ormai prossimo alla piena romanizzazione anche linguistica.

\subsubsection{Tavola dei caratteri}

Modello alfabetico d'uso
A 8
$习 \supset * \frac{日}{\theta}$
$1 \times \mathrm{JHH}$
1
$02 \times v$
$89 d$

Trascrizione:

$a b$

e $v \quad z h$

$\begin{array}{lllll}i & k & l & m & n\end{array}$

$\begin{array}{lllll}p & r & s & t & u\end{array}$

$f \ddot{r} \xi$

Val. fonetico:

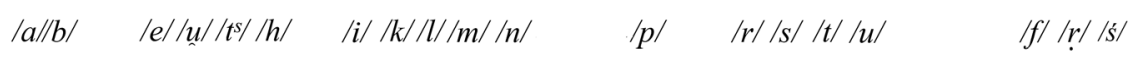

\subsubsection{Esempi illustrativi}

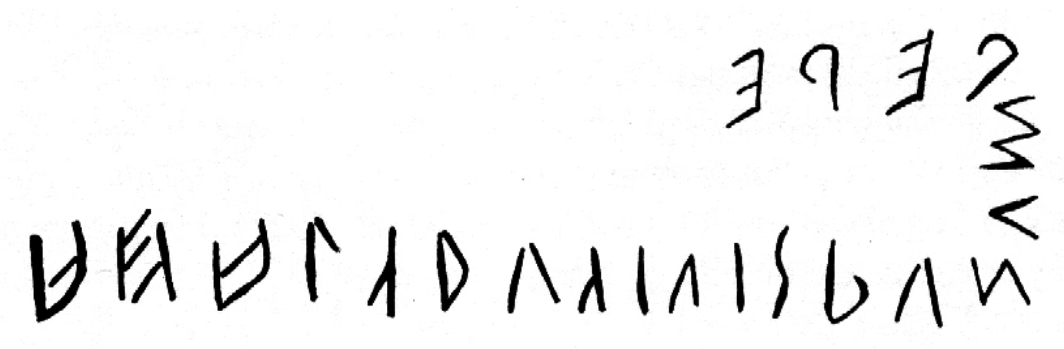

Fig. 17. Todi (TN). Statua bronzea, cd. «Marte di Todi» (fine del V sec. a.C.)

ahal trutitis řun/um / řrere

Bibl: Roncalli 1973, 91-101; Rocca 1996, 111, n. 19; ST Um 16; Morandi 2017, 135-136, n. 133.

50 Prosdocimi 1984; Agostiniani et alii 2011, 22, n. 17; Morandi 2017, 142-185, n. 139. 


\section{Questioni aperte e sfide per il futuro}

Come si è visto nelle pagine precedenti, anche se ci sono ormai relativamente pochi i problemi di decifrazione e lettura delle scritture epicoriche dell'Italia preromana, rimangono ancora numerosi aspetti da chiarire sullo sviluppo storico dei sistemi scrittori e sulle relazioni di interdipendenza tra i diversi modelli.

In particolare si possono individuare due grandi categorie di questioni sulle quali sarà impegnata la ricerca futura, a seconda se si tratti di problemi locali, interni a un singolo sistema scrittorio, ovvero di aspetti generali che investono le relazioni reciproche tra gruppi etno-linguistici diversi.

Alla prima categoria appartiene l'individuazione e lo studio di modelli alfabetici alternativi o concorrenti all'interno di specifici domini epigrafici sia a scala cittadina che regionale. Un esempio significativo in questo senso è la definizione delle scritture latine 'extra-urbane' in epoca arcaica, resa difficile dalla carenza di documentazione, ridotta a qualche decina di iscrizioni e frustuli, a fronte di un discreto numero di documenti epigrafici da Roma. ${ }^{51}$

Per converso, laddove è stato possibile far uso di una base statistica adeguata - come ad esempio nei centri dell'Etruria meridionale o nell'area Golasecchiana - l'analisi dettagliata dei modelli alfabetici d'uso ha consentito di riconoscere diverse tradizioni in opera, nello spazio e nel tempo. ${ }^{52}$

Questioni di cronologia e sviluppo storico sono ancora aperte per quanto riguarda le varie tradizioni grafiche paleo-italiche e il loro rapporto con la scrittura paleo-sabellica, evidentemente appartenenti a un unico filone epigrafico, ma ampiamente articolato e diffuso su scala inter-regionale. ${ }^{53}$

Un'analogo aspetto da investigare concerne le scritture dell'Italia settentrionale, con particolare riguardo al dominio celtico golasecchiano e a quello veneto, che presentano alcune convergenze nel corso dei secoli, pur mantenendo le proprie precise individualità. Del pari restano da approfondire le relazioni di questi due gruppi maggiori con quelli retico e camuno e con altri scarsamente documentati dell'arco alpino. ${ }^{54}$

51 Maras 2009b; 2009c; Mancini 2011; Muscariello 2015.

52 Gambari 2012; Maras 2014a.

53 Rocca 1997; Colonna 1999; Agostiniani 2010; Poccetti 2010; A. Calderini, in Agostiniani et al. 2011, 8-9.

54 Rubat Borel 2005; Eska e Wallace 2011; Marchesini 2011; 2014. 
In questo contesto, resta di primaria importanza il riconoscimento e lo studio diacronico e storico-archeologico dei contesti culturali in cui hanno operato le scuole scrittorie, i cui rapporti hanno presumibilmente determinato la maggior parte delle influenze reciproche che si osservano nei documenti scritti.

Da tale studio ci si può attendere un chiarimento dei rapporti osservati tra segni analoghi per forma e valore fonetico anche a grande distanza geografica e cronologica, come si è accennato per il digamma uncinato e per il segno ad alberello, ma anche per altri elementi grafici, come la $<o>$ bicaudata, i segni per le vocali intermedie e l'interpunzione sillabica. In altre parole, si tratta di ricostruire i canali di comunicazione culturale inter-etnica, attraverso i quali è stato possibile effettuare la trasmissione di singoli elementi dei modelli alfabetici e dei sistemi scrittori.

Questioni simili ed altre derivanti da nuove scoperte epigrafiche impegneranno il lavoro dei ricercatori nei prossimi anni, rendendo quanto mai dinamica e provvisoria la conoscenza delle scritture epicoriche d'Italia che si è tentato di riassumere in queste poche pagine. L'augurio dell'autore è pertanto di aver saputo almeno comunicare la varietà e articolazione delle tradizioni grafiche e la complessità delle sfide che si pongono di fronte agli studiosi di questo affascinante campo di ricerca.

\section{$\begin{array}{llllllllllll}\mathbf{B} & \mathbf{I} & \mathbf{B} & \mathbf{L} & \mathbf{I} & \mathbf{O} & \mathbf{G} & \mathbf{R} & \mathbf{A} & \mathbf{F} & \mathbf{I} & \mathbf{A}\end{array}$}

Agostiniani 2010: L. Agostiniani, "La nuova iscrizione di Sorrento e la definizione di paleoitalico in Italia e in Sicilia", in: F. Senatore e M. Russo (ed.), Sorrento e la Penisola Sorrentina tra Italici, Etruschi e Greci, Atti della giornata di Studio (Sorrento, 19 maggio 2007), Roma 2010, 57-63.

Agostiniani et al. 2011: L. Agostiniani, A. Calderini e R. Massarelli (ed.), Screhto est. Lingua e scrittura degli antichi Umbri, Perugia 2011.

Ammirati, Biagetti e Radiciotti 2006: S. Ammirati, C. Biagetti e P. Radiciotti, "Storia e geografia dell'alfabetismo in Grecia. Alle origini di un fenomeno", Studi di Egittologia e Papirologia 3, 2006, 9-30.

Ancillotti e Calderini 2009: A. Ancillotti e A. Calderini (a cura di), L'umbro e le altre lingue dell'Italia mediana antica, Atti del I Convegno Internazionale sugli Antichi Umbri (Gubbio, 2001), Perugia 2009.

Ancillotti e Cerri 1996: A. Ancillotti e R. Cerri, (Le Tavole di Gubbio e) La civiltà degli Umbri, Perugia 1996. 
Attenni e Maras 2004: L. Attenni e D. F. Maras, "Materiali arcaici dalla collezione Dionigi di Lanuvio ed il più antico alfabetario latino", SE 70, 2004, 61-78.

Bagnasco Gianni 1993: G. Bagnasco Gianni, "A proposito di tre kyathoi in bucchero a rilievo", in: Produzione artigianale ed espansione nel mondo antico. Il bucchero etrusco, Milano 1993, 207-216.

Bagnasco Gianni 1996: G. Bagnasco Gianni, Gli oggetti iscritti di epoca orientalizzante, Firenze 1996.

Bagnasco Gianni 1999: G. Bagnasco Gianni, "Lacquisizione della scrittura in Etruria: materiali a confronto per la ricostruzione del quadro storico e culturale", in: G. Bagnasco Gianni e F. Cordano (ed.), Scritture mediterranee tra il IX e il VII secolo a. C., Milano 1999, 85-106.

Bagnasco Gianni 2000a: G. Bagnasco Gianni, "La scrittura", in: M. Torelli (ed.), Gli Etruschi, Catalogo della mostra (Venezia, 2000), Milano 2000, 477-483.

Bagnasco Gianni 2000b: G. Bagnasco Gianni, "Lalfabeto etrusco", in: M. Negri (ed.), Alfabeti. Preistoria e storia del linguaggio scritto, Colognola ai Colli (VR) 2000, 151-176.

Bagnasco Gianni 2008: G. Bagnasco Gianni, “Comunicare per immagini: una questione di alfabeto", Aristhonotos 3, 2008, 47-72.

Bakkum 2009: G. Bakkum, The Latin Dialect of the Ager Faliscus: 150 years of scholarship, Amsterdam 2009.

Bartoněk e Büchner 1995: A. Bartoněk e G. Büchner, "Die ältesten griechischen Inschriften von Pithekoussai (2. Hälfte des VIII. bis 1. Hälfte des VII Jhs.)“, Die Sprache 37, 1995 , 129-231.

Bellelli 2008: V. Bellelli, “"Setums mi fece». Elementi per un riesame del cratere iscritto del Ferrone", in: P. Santoro (ed.), Una nuova iscrizione da Magliano Sabina. Scrittura e cultura nella valle del Tevere, Pisa-Roma 2008, 59-69.

Benelli 2001: E. Benelli, “The Romanization of Italy through the epigraphic record”, in: N. Terrenato (ed.), Italy nd the West. Comparative Issues in Romanization, Oxford 2001, 7-16.

Benelli 2004: E. Benelli, “Alfabeti greci e alfabeti etruschi”, AnnFaina 11, 2004, 291-305.

Bernardi Perini 1983: G. Bernardi Perini, "Le «riforme» ortografiche latine di età republicana", AIONLing 5, 1983, 141-169.

Biella 2020: M. C. Biella, “Drawing' Inscriptions: Preliminary Remarks on Writing Artisans in $7^{\text {th }}$ Century BC Faliscan Territory", in: R. D. Whitehouse (ed.), Etruscan in its Social Context, Oxford 2020, 91-102.

Blanck 1992: H. Blanck, Das Buch in der Antike, München 1992.

Briquel e Lejeune 1989: D. Briquel e M. Lejeune, Lingue e scritture, in: Italia omnium terrarum parens, Milano 1989, 433-474.

Bruschetti et al. 2015: P. Bruschetti, F. Gaultier, P. Giulierini, L. Haumesser e L. Pernet (ed.), Gli Etruschi maestri di scrittura. Società e cultura nell'Italia antica. Catalogo della mostra (Cortona-Montpellier, 2015-2016), Milano 2015, 25-37.

Calderini 2012: A. Calderini, "Aspetti linguistici delle iscrizioni presannitiche dell'area aurunca", in: U. Zannini (ed.), Isti (Aurunci) Graece Ausones nominantur. Atti del Convegno (Sessa Aurunca, 2009), Minturno 2012, 225-252.

Calderini, Neri e Ruggeri 2007: A. Calderini, S. Neri e M. Ruggeri, L'iscrizione sul "Guerriero di Capestrano (ST Sp AQ 2), in: M. Ruggeri (ed.), Guerrieri e re dell'Abruzzo antico, Ascoli Piceno 2007, 46-47. 
Camporeale 2015: G. Camporeale, Larrivo dellalfabeto in Etruria, in: P. Bruschetti, F. Gaultier, P. Giulierini, L. Haumesser e L. Pernet (ed.), Gli Etruschi maestri di scrittura. Società e cultura nell'Italia antica. Catalogo della mostra (Cortona-Montpellier, 2015-2016), Milano 2015, 18-23.

Cappuccini 2018: L. Cappuccini, "Un kyathos di bucchero da Poggio Pelliccia, la «bottega vetuloniese» e il suo ruolo nella trasmissione della scrittura in Etruria”, SE 80, 2018, 61-82.

CIE: Corpus Inscriptionum Etruscarum, Berlin-Roma.

Colonna 1970: G. Colonna, "Una nuova iscrizione etrusca del VII secolo e appunti sull'epigrafia ceretana dell'epoca", MEFRA 82, 1970, 637-672.

Colonna 1976: G. Colonna, "Il sistema alfabetico", in: Letrusco arcaico, Atti del colloquio (Firenze, 1974), Firenze 1976, 1-24.

Colonna 1980: G. Colonna, "Laspetto epigrafico", in: Lapis Satricanus, 's-Gravenhage 1980, 41-69.

Colonna 1984: G. Colonna, "Un trofeo di Novio Fannio comandante sannita", in: Studi di antichità in onore di G. Maetzke, II, Roma 1984, 229-241.

Colonna 1987: G. Colonna, "Note preliminari sui culti del santuario di Portonaccio a Veio", ScAnt 1, 1987, 419-446.

Colonna 1988a: G. Colonna, L'iscrizione, in: F. M. Gambari e G. Colonna, "Il bicchiere con iscrizione arcaica da Castelletto Ticino e l'adozione della scrittura nell'Italia nordoccidentale", SE 54, 1988, 130-164.

Colonna 1988b: G. Colonna, "Lécriture dans l'Italie centrale à l'époque archaïque", Revue de la Société des élèves, anciens élèves et amis de la section des sciences religieuses de l’É.P.H.É., 1988, 12-31.

Colonna 1991: G. Colonna, "Il posto dell'Arringatore nell'arte etrusca di età ellentistica", SE 56, 1991, 99-122.

Colonna 1994: G. Colonna, "Le iscrizioni di Nocera e il popolamento pre- e paleosannitico della valle del Sarno", in: A. Pecoraro (ed.), Nuceria Alfaterna e il suo territorio, Nocera Inferiore 1994, 85-99.

Colonna 1995: G. Colonna, "Etruschi a Pithecusa nellorientalizzante antico", in: Lincidenza dellantico. Studi in memoria di Ettore Lepore, Atti del convegno internazionale (Anacapri, 24-28 marzo 1991), Napoli 1995, 325-342.

Colonna 1999: G. Colonna, "L'iscrizione del biconico di Uppsala: un documento del paleoumbro", in: Incontro di studi in memoria di Massimo Pallottino, Pisa-Roma 1999, 19-29.

Colonna 2001: G. Colonna, "Gli Umbri del Tevere", Annali della fondazione per il Museo C. Faina di Orvieto 8, 2001, 9-30.

Colonna 2005: G. Colonna, L'iscrizione di Osteria dell'Osa, in: G. Bartoloni e F. Delpino (ed.), Oriente e Occidente: metodi e discipline a confronto, Riflessioni sulla cronologia delletà del Ferro italiana, Atti dell'incontro di studio (Roma, 30-31 ottobre 2003), Roma 2005, 479-483.

Colonna 2014: G. Colonna, "Il graffito di Villa Altieri sull'Esquilino: la più antica iscrizione da Roma (e un'altrimenti sconosciuta area sacra)", in: G. Baldelli e F. Lo Schiavo (ed.), Amore per l'antico. Dal Tirreno all'Adriatico, dalla Preistoria al Medioevo e oltre. Studi di antichità in ricordo di Giuliano de Marinis, Roma 2014, 15-23.

Colonna e De Simone 1983: G. Colonna, C. de Simone, “Un’iscrizione paleoitalica dallagro tolfetano", SE 51, 1983, 573-594.

Colonna e Gatti 1992: G. Colonna e S. Gatti, Anagni. Iscrizioni in dialetto ernico, SE 58, 1992, 320-327. 
Cooley 2002: A.E. Cooley, “The survival of Oscan in Roman Pompeii”, in: A. E. Cooley (ed.). Becoming Roman, Writing Latin? Literacy and Epigraphy in the Roman West, Portsmouth (RI), 2002, 77-86.

Cornell 1991: T. Cornell, "The Tyranny of the Evidence. A Discussion of the Possible Uses of Literacy in Etruria and Latium in the Archaic Age", in: J. H. Humphrey (ed.), Literacy in the Roman World, Ann Arbor (MI) 1991, 7-33.

Crawford 2011: M. H. Crawford, Imagines Italicae: A Corpus of Italic Inscriptions, 1-3, London 2011.

Cristofani 1969: M. Cristofani, “Appunti di epigrafia etrusca arcaica”, AnnPisa 38, 1969, 99-113.

Cristofani 1972: M. Cristofani, "Sull'origine e la diffusione dell'alfabeto etrusco", ANRW 1/2, Berlin 1972, 466-489.

Cristofani 1982: M. Cristofani, Contatti fra Lazio ed Etruria in età arcaica: documentazione archeologica e testimonianze epigrafiche, in: Alle origini del latino. Atti del Convegno della SIG (Pisa, 7-8 dicembre 1980), Pisa 1982, 27-42.

Cristofani 1990: M. Cristofani, "La formazione della scrittura in Etruria”, AnnFaina 4, 1990, 61-73.

Cristofani 1996: M. Cristofani, "Per Regna Maricae, in Due testi dell'Italia preromana", Quaderni di Archeologia Etrusco-Italica, 25, Roma 1996, 9-32.

Del Tutto Palma 1996: L. Del Tutto Palma (ed.), La Tavola di Agnone nel contesto italico, Atti del convegno (Agnone, 13-15 aprile 1994), Firenze 1996.

De Marinis et al. 2009: R. C. de Marinis, S. Massa e M. Pizzo (ed.), Alle origini di Varese e del suo territorio: le collezioni del sistema archeologico provinciale, Roma 2009.

De Marinis e Motta 1990-1991: R. C. de Marinis e F. Motta, "Una nuova iscrizione lepontica su pietra da Mezzovico (Lugano)”, Sibrium 21, 1990-1991, 201-225.

De Simone 1992: C. de Simone, "Sudpiceno Safino- / latino Sabino-: il nome dei Sabini", AIONLing. 14, 1992, 223-239.

De Simone 2006: C. de Simone, "Liscrizione "aurunca" del Garigliano: nuove considerazioni critiche", in: R. Bombi, G. Cifoletti, F. Fusco, L. Innocente e V. Orioles (ed.), Studi linguistici in onore di Roberto Gusmani, Alessandria 2006, 549-575.

De Simone e Marchesini 2013: C. de Simone e S. Marchesini, La lamina di Demlfeld, Pisa-Roma 2013.

Eska e Wallace 2011: J. F. Eska e R. E. Wallace, "Script and Language at Ancient Voltino", in: Le lingue dell'italia antica: iscrizioni, testi, grammatica, Atti del Convegno in memoria di Helmut Rix (Milan, 2011), Alessandria 2012, 93-113.

ET: H. Rix (coord.), Etruskische Texte, Tübingen 1991.

Farney 2011: G. D. Farney, "Aspects of the Emergence of Italian Identity in the Early Roman Empire", in: M. Gleba e H. W. Horsnaes (edd.), Communicating identity in Italic Iron Age communities. Oxford-Oakville 2011, 223-232.

Fortini 2005: P. Fortini, "Una nuova iscrizione latina arcaica dal Foro Romano (area del cd. Equus Domitiani)", in: D. Caiazza (ed.), Italica ars. Studi in onore di G. Colonna per il premio I Sanniti, Piedimonte Matese 2005, 267-276.

Gambari 2012: F. M. Gambari, La scrittura dei Celti golasecchiani, in: Le grandi vie delle civiltà. Relazioni e scambi fra Mediterraneo e il centro Europa dalla preistoria alla romanità, Catalogo della mostra (Trento, 2011-2012), Trento 2011, 393-394.

Giacomelli 1963: G. Giacomelli, La lingua falisca, Firenze 1963.

Giacomelli 2006: G. Giacomelli, Nuove ricerche falische, Roma 2006. 
Guzzo 2011: P. G. Guzzo, “Sul graffito dalla necropoli dell'Osteria dell’Osa”, Rivista dell'Istituto Nazionale di Archeologia e Storia dell'Arte 61, 2011, 63-66.

Harris 1989: W. V. Harris, Ancient Literacy, Cambridge (MA)-London 1989.

Hartmann 2005: M. Hartmann, Die frühlateinischen Inschriften und ihre Datierung, Bremen 2005.

Johnston 1983: A. Johnston, “The Extent and Use of Literacy: the Archaeological Evidence”, in: R. Hagg (ed.), The Greek Rennaissance of the eighth century BC: tradition and innovation, Stockholm 1983, 63-68.

La Regina 2010: A. La Regina, "Il Guerriero di Capestrano e le iscrizioni paleosabelliche”, in: L. Franchi dell'Orto (ed.), Pinna Vestinorum I, Roma 2010, 230-273.

Lazzarini 1999: M. L. Lazzarini, "Questioni relative allorigine dellalfabeto greco", in: G. Bagnasco Gianni e F. Cordano (ed.), Scritture mediterranee tra il IX e il VII secolo a. C., Milano 1999, 53-66.

Lazzarini e Poccetti 2001: M. L. Lazzarini e P. Poccetti, “L'iscrizione paleoitalica da Tortora”, in: M. Bugno e C. Masseria (ed.), Il mondo enotrio tra VI e V secolo a.C. Atti dei seminari napoletani (1996-1998), Napoli 2001 .

Lejeune 1957: M. Lejeune, "Sur l'adaptation de l'alphabet étrusque aux langues indoéuropéennes", REL 35, 1957, 88-105.

Lejeune 1971: M. Lejeune, Lepontica, Paris 1971

Lejeune 1974: M. Lejeune, Manuel de la langue vénète, Heidelberg, 1974.

Leszkiewicz 2017: B. Leszkiewicz, Język Faliski, Kraków 2017.

Maggiani 1990: A. Maggiani, "Alfabeti etruschi di età ellenistica”, AnnFaina 4, 1990, 177-217.

Maggiani 2014: A. Maggiani, "Un kyathos di bucchero da Tolle (Chiusi)”, in: S. Bruni (ed.), 'Lautus erat Tuscis Porsena fictilibus'. Studi e ricerche sul bucchero dell'area chiusina per Luigi Donati, Pisa 2014, 19-39.

Maggiani 2018: A. Maggiani, "La nascita della scrittura nell'Etruria settentrionale. Una nota", SE 80, 2018, 133-147.

Mancini 1980: A. Mancini, "Le iscrizioni della Valcamonica", in: Studi urbinati di storia, filosofia e letteratura. Suppl. linguistico 2, Urbino 1980, 75-166.

Mancini 1999: A. Mancini, "Iscrizioni retiche: aspetti epigrafici”, in: G. Ciurletti e F. Marzatico (ed.), I Reti / Die Räter, Trento 1999, 297-333.

Mancini 2004: M. Mancini, "Latina Antiquissima I: esercizi sulla Fibula Praenestina”, Daidalos 6, 2004, 1-30.

Mancini 2011: M. Mancini, "Scritture e lingue nel Lazio protostorico e nell'Ager Faliscus: un bilancio", AIONLing 30, 2011, 193-297.

Mangani 2015: E. Mangani (ed.), Nuove ricerche sulla Fibula Prenestina, Atti della Giornata di Studi (Roma, 2011), Roma 2011-2014 (2015).

Maras 2005: D. F. Maras, "L'iscrizione di Trivia ed il culto del santuario alla foce del Garigliano", Archeologia Classica 56, n.s. 6, 2005, 33-48.

Maras 2009a: D. F. Maras, “Interferenze culturali arcaiche etrusco-latine: la scrittura”, AnnFaina $16,2009,309-331$.

Maras 2009b: D. F. Maras, "Novità sulla diffusione dell'alfabeto latino nel Lazio arcaico", in: F. Mannino, M. Mannino e D. F. Maras (ed.), Theodor Mommsen e il Lazio antico, Atti della Giornata di Studi (Terracina, 3 aprile 2004), Roma 2009, 105-118. 
Maras 2009c: D. F. Maras, “Caratteri dell'epigrafia latina arcaica del Lazio meridionale”, in: L. Drago Troccoli (ed.), Il Lazio dai Colli Albani ai Monti Lepini tra preistoria ed età moderna, Roma 2009, 431-439.

Maras 2009d: D. F. Maras, Il dono votivo. Gli dei e il sacro nelle iscrizioni etrusche di culto, PisaRoma 2009.

Maras 2012a: D. F. Maras, La scrittura dei principi etruschi, in: Etruschi. L'ideale eroico e il vino lucente, Catalogo della mostra (Asti, marzo-luglio 2012), Milano 2012, 103-109.

Maras 2012b: D. F. Maras, "Skerfs", in: Le lingue dell'italia antica: iscrizioni, testi, grammatica, Atti del Convegno in memoria di Helmut Rix (Milan, 2011), Alessandria 2012, 185-197.

Maras 2012c: D. F. Maras, "La tradizione grafica delle iscrizioni presannitiche dell'area aurunca", in: U. Zannini (ed.), Isti (Aurunci) Graece Ausones nominantur. Atti del Convegno (Sessa Aurunca, 2009), Minturno 2012, 219-224.

Maras 2013a: D. F. Maras, "Interferenza e concorrenza di modelli alfabetici e sistemi scrittori nell'Etruria arcaica", in: G. Van Heems e L. Haumesser (eds.), Régler l'usage: norme et standard dans l'Italie préromaine. Actes de les Rencontres (Rome, 2008-2011), Roma 2013, 331-344.

Maras 2013b: D. F. Maras, "Questioni di identità: Etruschi e Falisci nellagro falisco", in: G. Cifani (ed.), Identità e cultura dei Falisci. Atti del seminario presso la British School at Rome (Roma, 2011), Roma 2013, 265-285.

Maras 2014a: D. F. Maras, "Breve storia della scrittura celtica d'Italia. L'area Golasecchiana", Zixu 1, 2014, 73-93.

Maras 2014b: D. F. Maras, "Principi e scribi: alle origini dellepigrafia leponzia”, in: B. Grassi e M. Pizzo (ed.), Gallorum Insubrum fines, Atti della Giornata di Studi (Varese, 2010), Roma 2014, 101-109.

Maras 2015: D. F. Maras, "Etruscan and Italic Literacy and the Case of Rome", in: W. M. Bloomer (ed.), A Companion to Ancient Education, San Francisco 2015, 201-225.

Maras 2016: D. F. Maras, "Lettere e sacro. Breve storia della scrittura nel santuario etrusco di Pyrgi”, Studi Epigrafici e Linguistici sul Vicino Oriente Antico 32-33, 2016, 89-101.

Maras 2017: D. F. Maras, "Epigraphy \& Nomenclature”, in: G. Bradley e G. Farney (edd.), The Peoples of Ancient Italy, Berlin-Boston 2017, 59-84.

Maras 2020: D. F. Maras, “Traces of Orality in Writing”, in: R. D. Whitehouse (ed.), Etruscan Literacy in its Social Context, Oxford 2020, 125-134.

Maras c. s.: D. F. Maras, "Lepigrafia delle corti aristocratiche di Caere in epoca orientalizzante", in: L. Haumesser (ed.), Cerveteri. La culture écrite d'une cité étrusque, Actes du colloque (Paris, 2016), Paris in corso di stampa.

Marchesini 2011: S. Marchesini, "Alla ricerca del modello perduto. Sulla genesi dell'alfabeto camuno", Palaeohispanica 11, 2011, 155-171.

Marchesini 2014: S. Marchesini, "Nuove iscrizioni retiche da Cles e Sanzeno (TN)", in: R. Roncador e F. Nicolis (ed.), Antichi popoli delle Alpi. Sviluppi culturali durante l'età del Ferro nei territori alpini centro-orientali, Trento 2014, 127-144.

Marchesini 2015: S. Marchesini (in collaborazione con R. Roncador), Monumenta Linguae Raeticae, Roma 2015.

Marinetti 1985: A. Marinetti, Le iscrizioni sudpicene, I. Testi, Firenze 1985.

Marinetti 2002: A. Marinetti (ed.), AKEO. I tempi della scrittura. Veneti antichi: alfabeti e documenti, Catalogue of the exhibition (Montebelluna, December 2001- May 2002), Cornuda 2002.

Meiser 1987: G. Meiser, “Pälignisch, Latein und Südpikenisch”, Glotta 65, 1987, 104-125. 
Minetti 2008: A. Minetti, "Il biconico di Uppsala", in: P. Santoro (ed.), Una nuova iscrizione da Magliano Sabina. Scrittura e cultura nella valle del Tevere Roma 2008 , 53-57.

Morandi 1999: A. Morandi, Il cippo di Castelciès nell'epigrafia Retica, Roma 1999

Morandi 2004: A. Morandi, “Epigrafia e lingua dei Celti d'Italia”, in: P. Piana Agostinetti (ed.), Celti d'Italia, Roma 2004, vol. 2.

Morandi 2009: A. Morandi, "Minturno. Santuario di Marica. Iscrizione cosiddetta di "Trivia”, LazioßSabina 5, 2009, 445-446.

Morandi 2017: A. Morandi, Epigrafia italica 2, Roma 2017.

Motta 2000: F. Motta, "La documentazione epigrafica e linguistica", in: R .C. de Marinis e S. Biaggio (ed.), I Leponti tra mito e realtà, Raccolta di saggi in occasione della mostra, 1-2, Locarno 2000.

Muscariello 2015: M. Muscariello, "Influenze sulla nascita della scrittura nel Latium vetus: il latino arcaico", in: E. Dupraz e W. Sowa (eds.), Genres épigraphiques et langues d'attestation fragmentaire dans l'espace méditerranéen, Rouen-Havre 2015, 47-67.

Pandolfini 2015: M. Pandolfini, "L'inizio della scrittura etrusca nell'epoca orientalizzante", in: P. Bruschetti, F. Gaultier, P. Giulierini, L. Haumesser e L. Pernet (ed.), Gli Etruschi maestri di scrittura. Società e cultura nell'Italia antica. Catalogo della mostra (CortonaMontpellier, 2015-2016), Milano 2015, 25-37.

Pandolfini e Prosdocimi 1990: M. Pandolfini e A. L. Prosdocimi, Alfabetari e insegnamento della scrittura in Etruria e nell'Italia antica, Firenze 1990.

Pellegrini e Prosdocimi 1967: G. Pellegrini, A. L. Prosdocimi, La lingua venetica, Padova 1967.

Poccetti 1979: P. Poccetti, Nuovi documenti italici a complemento del manuale di Emil Vetter, Pisa 1979.

Poccetti 2010: P. Poccetti, "Intorno ai nuovi documenti di area sorrentina: riflessioni sul novum e sul notum", in: F. Senatore e M. Russo (ed.), Sorrento e la Penisola Sorrentina tra Italici, Etruschi e Greci nel contesto della Campania antica, Atti della giornata di Studi (Sorrento, 19 maggio 2007), Roma 2010, 65-101.

Prosdocimi 1984: A. L. Prosdocimi, Le Tavole Iguvine, Firenze 1984.

Prosdocimi 1990: A. L. Prosdocimi, "Lalfabeto come insegnamento e apprendimento", AnnFaina 4, 1990, 113-175.

Prosdocimi 1991: A. L. Prosdocimi, "Note sul celtico in Italia", SE 57, 1991, 139-177.

Prosdocimi 2007: A. L. Prosdocimi, "Nota sulla convenzione grafica delle dentali nell'alfabeto venetico (REI, parte III)", SE 73, 2007, 461-473.

Prosdocimi 2009: A. L. Prosdocimi, "Sulla scrittura nell'Italia antica", in M. Mancini e B. Turchettta (a cura di), Scrittura e scritture: le figure della lingua. Atti del Convegno (Viterbo, 28-30 ottobre 2004), Roma 2009, 143-231.

Rigobianco 2019: L. Rigobianco, Faliscan. Language. Writing. Epigraphy, Zaragoza 2019.

Riva 2006: C. Riva, “The Orientalizing Period in Etruria: sophisticated communities”, in: C. Riva e N. C. Vella (eds.), Debating Orientalization. Multidisciplinary Approaches to Change in the Ancient Mediterranean, London 2006, 110-134.

Rix 1992: H. Rix, Thesen zum Ursprung der Runenschrift, in: Etrusker nördlich von Etrurien, Akten des Symposions (Wien, Schloß Neuwaldegg, 1989), Wien 1992, 411-441.

Rix 1996: H. Rix, "Il testo paleoumbro di Poggio Sommavilla", SE 41, 1996, 233-246.

Rix 1998a: H. Rix, Etruskisch und Rätisch, Innsbruck 1998.

Rix 1998b: H. Rix, "Eine neue frühsabellische Inschrift und der altitalische Präventiv", Historische Sprachforschung 1111, 1998, 245-269. 
Rix 2000: H. Rix, “Scrittura e lingua”, in: M. Cristofani (ed.), Gli Etruschi. Una nuova immagine, Firenze $2000^{2}, 199-227$.

Rix 2009: H. Rix, "Umbro e sudpiceno: differenze e concordanze”, in: A. Ancillotti e A. Calderini (ed.), L'umbro e le altre lingue dell'Italia mediana antica, Atti del I Convegno Internazionale sugli Antichi Umbri (Gubbio, 2001), Perugia 2009, 249-264.

Rocca 1996: G. Rocca, Le iscrizioni umbre minori, Firenze 1996.

Rocca 1997: G. Rocca, "I rapporti del latino con le varietà italiche. Il caso di Satricum”, in: Nomen Latinum (Atti del Conv. Internaz., Roma 1995), Roma 1997, 189-198.

Rocca 2000: G. Rocca, “Gli alfabeti d'Italia”, in: M. Negri (ed.), Alfabeti. Preistoria e storia del linguaggio scritto, Colognola ai Colli (VR) 2000, 177-204.

Rocca 2001: G. Rocca, “The Poggio Sommavilla inscription”, in: M. E. Huld, K. Jones-Bley, A. Della Volpe e M. Robbins Dexter (eds.), Proceedings of the 12th Annual UCLA IndoEuropean Conference, Washington 2001, 107-131.

Roncalli 1973: F. Roncalli, "Il «Marte» di Todi. Bronzistica etrusca ed ispirazione classica”, in: MemPontAc, s. III, 11/2, 1973, 91-101

Roncalli 1985: F. Roncalli (ed.), Scrivere Etrusco, Catalogo della mostra (Perugia, 1985), Milano 1985.

Rubat Borel 2005: F. Rubat Borel, "Lingue e scritture delle Alpi occidentali prima della romanizzazione. Stato della questione e nuove ricerche", BEPA 16, 2005, 9-50.

Russo 2005: M. Russo, Sorrento. Una nuova iscrizione paleoitalica in alfabeto 'nucerino', Capri 2005.

Salomon 2017: C. Salomon, Rético. Lengua. Escritura. Epigrafía, Zaragoza 2017.

Sassatelli 2000: G. Sassatelli, "Le iscrizioni della cultura di Golasecca", in: M. A. Binaghi e M. Squarzanti (ed.), Museo civico di Sesto Calende. La raccolta archeologica e il territorio, Gallarate 2000, 50-57.

Schumacher 1992: S. Schumacher, Die rätischen Inschriften, Innsruck 1992.

Schumacher 1998: S. Schumacher, „Sprachliche Gemainsamkeiten zwischen Rätisch und Etruskisch“, Der Schmern, 72/2, 1998, 90-114.

Schumacher 2004: S. Schumacher, Die rätische Inschriften, 2. erweiterte Auflage (Innsbrucker Beiträge zur Kulturwissenschaft, 79. Sonderheft), Innsbruck 2004.

Sisani 2009: S. Sisani, Umbrorum gens antiquissima Italiae, Perugia 2009.

Solinas 1995: P. Solinas, "Il celtico in Italia", SE 60, 1995, 311-408.

Solinas 2002: P. Solinas, "Spie di ideologia etnica in epigrafi celtiche di area veronese. Tra grafia e cultura", SE 65-68, 2002, 275-298.

ST: H. Rix (Hrsg.), Sabellische Texte. Die Texte des Oskischen, Umbrischen und Südpikenischen, Heidelberg 2002.

Stoklund 1997: M. Stoklund, "Le rune", in :Riflessi di Roma. Impero Romano e barbari nel Baltico (Cat. della Mostra, Milano, 1 marzo-1 giugno 1997), Roma 1997, 112-114

Tagliamonte 2010: G. Tagliamonte, “L'iscrizione CIE 8806 da Vico Equense”, in: F. Senatore e M. Russo (ed.), Sorrento e la Penisola Sorrentina tra Italici, Etruschi e Greci nel contesto della Campania antica. Atti della giornata di Studi (Sorrento, 19 maggio 2007), Roma 2010, 103-121.

Tibiletti Bruno 1990: M. G. Tibiletti Bruno, "Nuove iscrizioni camune”, Quaderni Camuni 4950, 1990, 29-171. 
Tikkanen 2020: K.W: Tikkanen, "Lost - and Found - in Transmission", in: J. Clackson, P. James, K. McDonald, L. Tagliapietra, N. Zair (eds.), Migration, Mobility and Language Contact in and around the Ancient Mediterranean, Cambridge 2020, 98-121.

Triantafillis 2008: E. Triantafillis, Le iscrizioni italiche dal 1979. Testi, retrospettiva, prospettive, Padova 2008.

Tuck e Wallace 2020: A. Tuck, R. E. Wallace, "The Social Context of Proto-Literacy in Central Italy: the Case of Poggio Civitate", in: R. D. Whitehouse (ed.), Etruscan Literacy in its Social Context, Oxford 2020, 9-20.

Untermann 2000: J. Untermann, Wörterbuch des Oskisch-Umbrischen, Heidelberg 2000.

Urbanovà 1999: D. Urbanovà, "La paleografia delle iscrizioni latine arcaiche", in: Atti XI Congresso Internazionale di Epigrafia Greca e Latina (Roma, 18-24 settembre 1997), Roma 1999, 477-492.

Verger 2001: S. Verger, "Un graffite archaïque dans l'habitat halstattien de Montmorot (Jura, France)", SE 64, 1998 (2001), 265-316.

Wallace 2004a: R. E. Wallace, “The Sabellian Languages”, in: R. D. Woodard (ed.), The Cambridge Encyclopedia of the World's Ancient Languages, Cambridge-New York-Melbourne 2004, 812-839.

Wallace 2004b: R. E. Wallace, "Venetic", in: R. D. Woodard (ed.), The Cambridge Encyclopedia of the World's Ancient Languages, Cambridge-New York-Melbourne 2004, 840-856.

Wallace 2007: R. E. Wallace, The Sabellic Languages of Ancient Italy, München 2007.

Wallace 2008: R. E. Wallace, Zikh Rasna. A Manual of the Etruscan Language and Inscriptions, Ann Arbor-New York 2008.

Wallace 2010: R. E. Wallace, "Alphabet, Orthography and Paleography at Poggio Civitate (Murlo)", Etruscan Studies 13, 2010, 109-122.

Wallace e Tuck 2011: R. E. Wallace e A. Tuck, "An inscribed rocchetto from Poggio Civitate (Murlo)", SE 74, 2011, 193-198. 